\title{
Consciousness-related interactions in a double-slit optical system
}

\author{
Gabriel Guerrer* \\ Instituto de Psicologia da Universidade de São Paulo, SP, Brasil
}

March 9, 2018

\begin{abstract}
Motivated by a series of reported experiments and their controversial results, the present work investigated if volunteers could causally affect an optical double-slit system by mental efforts alone. The participants' task in the experimental sessions alternated between intending an increase in the (real-time feedback-informed) amount of light diffracted through a specific single slit versus relaxing their intentional effort. In total, 240 sessions contributed by 171 volunteers were recorded. The first 160 sessions were collected in an exploratory mode, and those data revealed statistically significant differences between the intention and relax conditions. The analysis method and variables of interest derived from the exploratory sessions were then pre-registered for the subsequent 80 formal sessions. The formal experiments, based on a directional hypothesis, were not statistically significant. A post hoc meta-analysis based on a bi-directional hypothesis, and applied to the same data, resulted in a 2.75 sigma outcome $\left(p=6.02 \times 10^{-3}\right.$; es $\left.=0.31 \pm 0.2295 \% \mathrm{CI}\right)$. Directional and bidirectional analyses applied to an equal number of control sessions, all conducted without observers present, resulted in uniformly non-significant outcomes. Analysis of environmental factors did not reveal any artifactual sources that might have produced the significant bi-directional effect. While the pre-registered analysis did not support the existence of the investigated phenomenon, the post hoc findings warrant further investigation to formally test the bi-directional hypothesis.
\end{abstract}

\section{Introduction}

One of the hardest problems still unsolved by modern science concerns the nature of consciousness and its relationship to matter [1. The millennial old debate, currently addressed by the philosophy of mind, proceeds by asking if there are more fundamental aspects to reality, what are their properties, and how do they interact.

The first time physicists seriously considered the possibility of a role for consciousness within their discipline coincided with the development of quantum mechanics in the $20^{\text {th }}$ century. In particular, the question of how the superposition state is reduced to a definite observed state, known as the quantum measurement problem, led some scientists 22 4] to associate such abrupt transitions with an increase in subjective knowledge. According to that interpretation, the conscious agent played an essential role in promoting the state reduction when gaining information by interacting with the experimental apparatus.

The question evolved into a controversial philosophical and theoretical debate [5-11] where the majority today denies the necessity of any "extra-physical" consciousness ingredient in quantum physics [12. Data in support of the leading view is found in "which-path" experiments, reported and discussed by [13]. Those experiments reveal that a sufficient condition for a superposition state collapse is the availability of "which-path" information, even when theoretically

\footnotetext{
*guerrer@usp.br / gabrielguerrer@gmail.com
} 
obtainable but not effectively measured. The examples provided in 13 lead to the conclusion that information reaching human consciousness is not a mandatory step for state reduction.

Although a strong role for human consciousness in the quantum measurement problem may be ruled out, a weaker but theoretically highly significant role can be investigated; if the right conditions are met, can consciousness influence the collapse of the superposition state? Or, more generally, is there any sort of interaction between consciousness-related mental states and quantum systems?

Experimental efforts to address the above questions date back to the 1970's with the use of random number generators. These devices use quantum effects such as radioactive decay and tunneling to produce truly random binary numbers. In those studies, participants tried to directionally bias the 0 or 1 outcomes from 50/50 chance through their mental intention, usually being informed in real time about the measured values. Two major meta-analyses [14,15] have reported statistical evidence for the anomalous correlation between conscious intention and the output of random number generators. The results revealed a goal-oriented characteristic, where the increase in 0 or 1 coincides with the participant's intended aim. Although significant, [15] concluded that the effect could be more simply explained as an artifact attributable to nonsignificant unpublished studies. That interpretation was argued as insufficient by the authors of the first meta-analysis [16.

A double-slit system as a target in a similar experimental protocol was first used by [17. In the standard double-slit system if partial which-path information is obtained by any means, one expects a reduction in the interference component [18. That study investigated the fringe visibility (a measure of the interference component) variation according to the participant's intention. Two experiments were presented, one supporting the interaction hypothesis and the other conforming to chance expectations.

Of particular interest to the present work are the double-slit experiment series [19 22] presented by Dean Radin and his collaborators. Those results are remarkable in the sense that many of the pre-planned experiments resulted in statistically significant evidence supporting the investigated interaction. Their findings, across the work series, claim that the observed effects: a) globally support the mind-matter interaction hypothesis, i.e. the causal effect of the participant's intention in the optical system; b) cannot be explained as procedural or analytical artifacts, as the control sessions (without participants present) resulted in no significant differences between the intention-present and intention-absent epochs; c) are stronger for participants with contemplative practices training, e.g. meditation; d) show a positive correlation to the participant's score obtained in an absorption questionnaire [23], which measures the degree of immersion that one can reach when performing a task; e) show a positive correlation to $\alpha$-band desynchronization, a marker of a shift in attention as measured by an electroencephalogram; f) are retro-causal, i.e. obtainable even when the participant views previously recorded data that was not observed by any participant or the experimenter prior to the session; g) do not depend on distance, occurring even when the participant tries to exert influence on a distant optical system while receiving the feedback information about the state of the interference pattern streamed over the internet. As a result, the effect sizes obtained do not appear to decline with distance.

Inspired by Radin et al's challenges to the present scientific world view, the current experiment tried to replicate their first four findings using a similar protocol and a modified setup/analysis as described below. 


\section{Methods}

\subsection{Equipment}

A semiconductor laser diode $L$ (DL-3148-023, single mode $\lambda=635 \mathrm{~nm}$, transverse magnetic polarization; Sanyo) is powered through a feedback driver circuit to maintain a constant $3 \mathrm{~mW}$ light output power. To minimize temperature fluctuations, the laser diode is mounted on a metal structure covered with styrofoam. No lenses or neutral density filters are employed.

As depicted in Fig. (11), the laser light passes through two slits $D S$ etched in a metal foil (10 $\mu \mathrm{m}$ width each, centrally-separated by $200 \mu \mathrm{m}$; Lenox Laser). The resulting interference pattern is recorded at $10 \mathrm{~Hz}$ by a CCD camera $C$ (FL3-GE-13S2M-C, 1288 x 964 pixel, 3.75 $\mu \mathrm{m}$ pixel size, $47 \%$ quantum efficiency at $\lambda=635 \mathrm{~nm}, 12$-bit ADC; FLIR) running at room temperature with a heat sink attached to its top. An internal $1 \mathrm{~mm}$ width protective glass is removed from the camera to minimize refraction distortions.

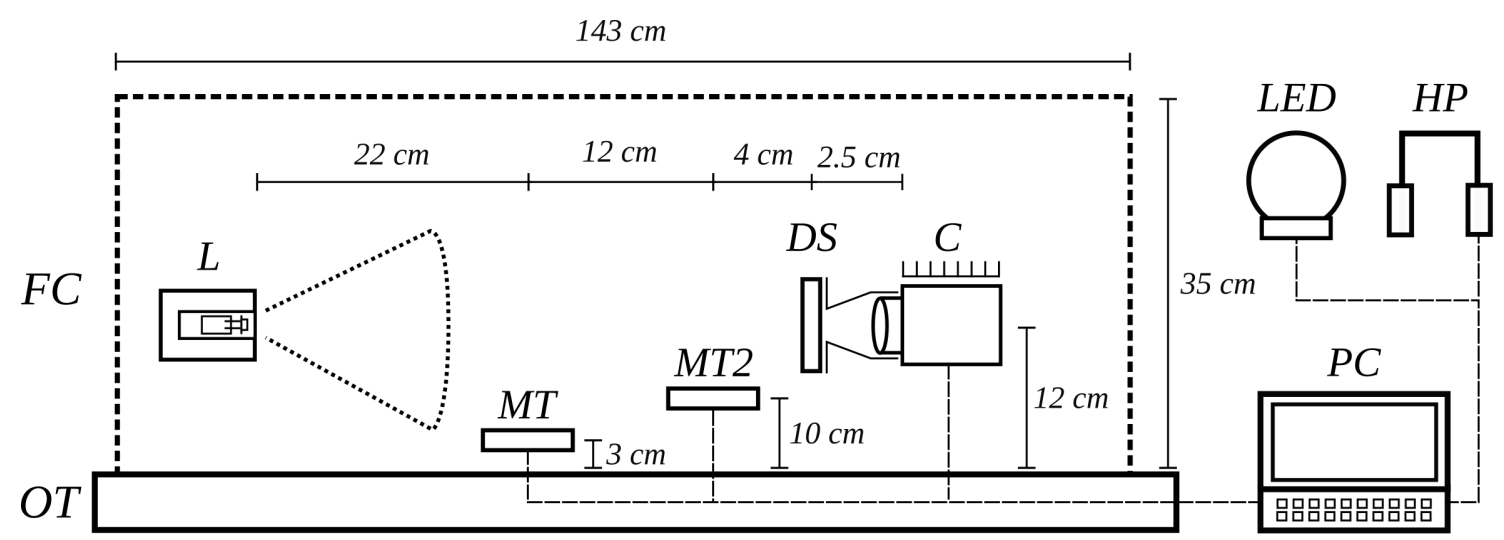

Figure 1: Experiment schematic side view. The distance of $2.5 \mathrm{~cm}$ represents the separation between the double-slit and the camera wall. The distance to the camera sensor is found with a fit procedure described in Section 2.6.

A 3D-printed hollow piece is used to connect the camera to the double-slit. One end is firmly attached to the camera's barrel and the other to a circular metallic piece that holds the double-slit foil. The plastic material color chosen is black to block light influences other than from the laser.

Concurrent with the CCD frame, temperature and magnetic field measurements are obtained using: a) an LM35 temperature sensor $\left(0.5^{\circ} \mathrm{C}\right.$ accuracy; Texas Instruments) coupled to the laser metal structure; b) an LM35 sensor placed between the laser and the double-slit for measuring room temperature ; c) an HMC5883L magnetometer (0.73 milli-gauss resolution, 12-bit ADC; Honeywell) placed close to the previous temperature sensor; d) an Arduino UNO microcontroller used to digitally read the sensors information. The whole system is presented as $M T$ in Fig. (1).

Starting at experiment 4, additional temperature and magnetic field measurements were obtained by the MT2 system consisting of: a) an LM35 temperature sensor coupled to the CCD heat dissipater; b) an HMC2003 magnetometer (0.04 milli-gauss analog resolution; Honeywell) placed close to the double-slit; c) a 4 channel 16-bit ADC (ADS1115; Texas Instruments); d) an Arduino UNO microcontroller.

The described components rest on a passively damped optical table OT (SmartTable UT; Newport) and are situated inside a grounded Faraday cage FC (tombak alloy, $82 \%$ copper and $18 \%$ zinc). The experiment is controlled by a $2 \mathrm{GHz}$ dual-core notebook computer $P C$ running a custom program developed in python language. Two devices are used to provide real-time feedback for the participants: noise canceling headphones HP (QuietComfort 25; Bose) and an Arduino controlled 3W LED placed inside a translucent glass sphere. The LED is composed of red, green, and blue color components that can be combined to produce a wide range of colors. 
A grounded uninterruptible power supply (Back-UPS 2200; APC) is used to feed the computer, the CCD camera and the laser power supply (MPS-3005; Minipa, Brazil) delivering $3.3 \mathrm{~V}$ DC to the driver circuit. The Arduino microcontrollers are powered through the $P C$ USB port. To ensure stable analog-to-digital (ADC) readings (concerning reference voltage variations), the following measures are taken at MT: a) the LM35 readings are obtained by the microcontroller 10-bit ADC using the regulated internal $1.1 \mathrm{~V}$ reference; b) The HMC5883L magnetometer is connected to a power regulated circuit module. At MT2, the LM35 and the HMC2003 are read by the ADS1115 ADC, which uses a regulated internal voltage reference.

\subsection{Data acquisition}

The python software controlling the experiment has its execution split into a two thread design. The first thread $T 1$ is a 10 frames per second loop responsible for simultaneously triggering the sensors readings and collecting the data output within each $100 \mathrm{~ms}$ window. The second thread $T 2$ represents the experiment flow, informing the participant about their current task, providing feedback depending on the current experimental condition, and performing data storage. As the program starts, $T 1$ is set to continuously acquire data while $T 2$ is in standby mode waiting for the command to start an experimental session. As a session starts, data arrays are sequentially filled with the sensors information captured by $T 1$. As the session ends, the data arrays are sent to hard-disk storage while $T 1$ continues its loop and $T 2$ returns to standby mode.

The CCD camera is configured to acquire frames using a $25 \mathrm{~ms}$ exposure time. Gain increase, auto-exposure and all post-processing filters (e.g. gamma, sharpness, brightness) are disabled. Each frame is initially obtained in a $1264 x$ per $256 z$ (centrally aligned) pixel window. Next, for every $x$, the $256 z$ values are summed, and the result is right bit shifted by 4 units. This oversampling technique, physically viable according to the $z$-axis system symmetry, is used to increase the measurement resolution from 12 to 16 bits. The resulting $1264 x$ values, referred to as a "CCD frame" throughout this work, represent the stored information used for the realtime feedback and the posterior analysis. Additionally, the temperature of the camera's internal components is obtained from an on-board temperature sensor $\left(0.5^{\circ} \mathrm{C}\right.$ accuracy; 12 -bit $\left.\mathrm{ADC}\right)$. Figure 2 shows an example of a single frame obtained with the current experimental setup and the interference pattern measured, as well as its Fourier transform components.

The HMC5883L sensor is configured to 8 averaged measurements per sample, and its gain is set to 0.73 milli-gauss resolution. In $M T$ all sensors are oversampled to reach 13-bit resolution (4 reads in HMC5883L and 64 in the LM35). In MT2 one single-ended ADS1115 reading (configured to a full scale-range of $\pm 4.096 \mathrm{~V}$ ) is performed for each sensor, resulting in an effective 15-bit resolution.

A $n=0,1, \ldots, n_{f}$ frame session results in the following data: a) a three valued condition array $C[n]$ tagging each frame to the corresponding experimental state of intention, relax, or a state in-between; b) a run array $R[n]$ comprised of $0 \ldots 39$ integers uniquely identifying the intention/relax 300-frame blocks; c) a CCD frame array $I[n, i]$ with $i=0,1, \ldots, 1263$ and 16-bit integer values; d) the temperature arrays $T_{C}[n], T_{L}[n]$ and $T_{R}[n]$ (32-bit floating point values) corresponding respectively to the on-board CCD camera, the laser and the room temperature sensors; e) the three-direction magnetic field arrays $M_{x}[n], M_{y}[n], M_{z}[n]$ (32-bit floating-point values) obtained by the $M T$ system sensor; f) from experiment 4 forward, the 32-bit float arrays corresponding to the CCD external temperature $T 2_{C}[n]$ and magnetic field components $M 2_{x}[n]$, $M 2_{y}[n], M 2_{z}[n]$, obtained from the $M T 2$ system sensors.

\subsection{Procedure}

To avoid potential warm-up artifacts and ensure thermal equilibrium, the following measures are taken 2 hours before each day's first session: a) laser diode and environmental sensors are turned on. In order to accelerate the CCD camera warm-up curve, it is powered on throughout 

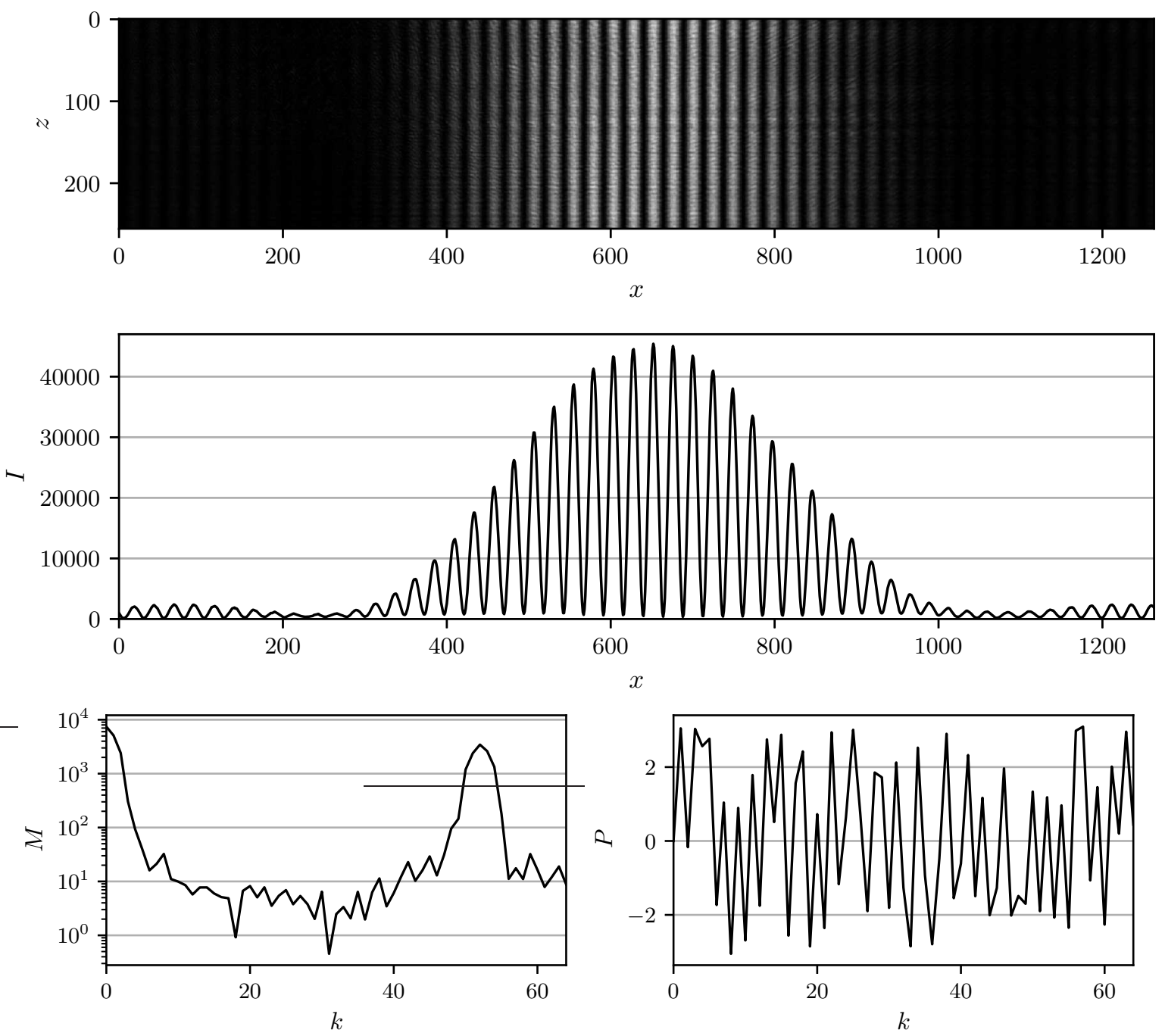

Figure 2: CCD frame information. Single raw-CCD frame showing the interference pattern measured (white representing the pixel brightness); 16-bit oversampled one-dimensional $I$ projection in analog digital units (the maximum value corresponds to $69 \%$ of the illumination capacity); and the $\log$-scale $M$ magnitude and $P$ phase components of the respective Fast Fourier Transform.

the entire experimental block to maintain its internal temperature even when in standby mode; b) the data acquisition software is started. Until the day's last session, the sensors will be uninterruptedly read at $10 \mathrm{~Hz}$; c) lights and air conditioning in the experimental room are switched off.

As the participant arrives at their scheduled time they sign an informed consent form describing the nature of the experiment. Next, they read a one-page text describing the task to be performed, and clarify any queries that they might have. Moving to the experimental room, the participant is briefly familiarized with the apparatus and the feedback devices. They sit in a chair about $3 \mathrm{~m}$ from the optical system, and are asked to remain seated and quiet during the entire session. They are then asked to put on the noise-canceling headphones. The experimenter switches the lights off, starts the session data acquisition, leaves the room, and waits for the session end in a nearby room. Shortly thereafter, over headphones, the participant hears a recorded message welcoming them, followed by guided instruction to take three deep 
breaths. The recording then announces the beginning of each test condition.

The volunteer's task alternates between two different conditions: intention and relax. In the first, they are asked to concentrate on the intention to increase the magnitude of the provided real-time feedback. The feedback system is designed to inform the participant about instantaneous variations in the amount of light crossing through a specific single-slit. By intending the feedback magnitude increase, the participant is indirectly attempting to enhance the number of photons passing through the feedback-targeted single-slit. During the relax condition, the participant stops receiving the feedback information, and is asked to temporarily cease any intention toward the experimental system.

Intention runs are announced with the phrase "prepare yourself", followed by a 3 -second silent delay, and then "... now, concentrate". The delay is included to facilitate the transition between an attention-away to an attention-toward mental state. Relax runs are announced with the phrase "now, relax". After the relax run ends, a random extra interval between 0 and 5 seconds is added to the in-between interval in order to decouple the measurements from possible periodic oscillations.

A single experimental session consists of 40 runs of alternating intention and relax conditions, with each run lasting $30 \mathrm{~s}$. Each session lasts about 28 minutes, and yields approximately 16,800 sensor data frames, of which 6,000 are obtained in the intention condition and 6,000 in the relax condition. The in-between data is comprised of the frames obtained during the welcome and the instructions playback, the 0-5 s random windows, and the additional $100 \mathrm{~s}$ collected after the last relax run. The tail data are important to absorb the polynomial-fit border artifacts.

After the session's end, the participant meets the experimenter in the next room. An automatic timer triggers a control session that starts 10 minutes later, running on the exact same computer code but with no person present in the experimental room. Before the control session start the experimenter ensures that the experimental room lights are off, and places the headphones on the chair. The same feedback LED colors and the same decoupling time delays of the previous participant session are used.

Considering the subjective nature of the task, the participants are requested to rely on their personal understanding of how they are to perform the task. However, two general guidelines are provided: a) they should try to avoid getting physically tired, thus acting in a present but detached way; b) they shouldn't expect to be able to exert absolute control on the feedback response. Given the random characteristics of the measurement the feedback is supposed to, under the null hypothesis, show unpredictable behavior. They are informed that their influence, if genuine, could be too small to be perceived. This information is important to help participants avoid any frustration during the session, and to promote a balanced state where, independent of the current feedback magnitude, the participant sustains a uniform intent.

During the sessions, the experimenter had no access to the current condition nor consciously tried to mentally influence the result. The data analysis was performed only at the end each pre-planned experimental block. Experimental sessions were scheduled on weekdays after $6 \mathrm{pm}$ and on Saturdays after $2 \mathrm{pm}$, and were separated by intervals of an hour and a half, usually allowing a maximum of three sessions during weekdays, and four on Saturdays.

The research was approved by the Comite de etica em pesquisa com seres humanos from Instituto de psicologia da Universidade de Sao Paulo, identifyied by CAAE 58223516.1.0000.5561.

\subsection{Hypothesis}

By intending the increase in the feedback magnitude, the participants are indirectly attempting to change the amount of light crossing each slit. In a standard double-slit experiment, the only way to causally promote such variation is by introducing into one of the slits some physical

agent to interact with the light. The proposed study extends the standard experiment by adding an extra component: a participant (also denominated as conscious agent) trying to mentally interact with the experimental system and influence the slits light intensity. According to the 
present scientific consensus, the agents must play a passive role, i.e. they shouldn't be able to modify the measured interference pattern with their introspective intentional efforts. Hence the null and the alternative hypothesis being tested are

$$
H_{0}:\left(\mu_{I}-\mu_{R}\right)=0 \quad ; \quad H_{1}:\left(\mu_{I}-\mu_{R}\right) \neq 0,
$$

where $\mu_{I}$ stands for the mean of measurements performed under the intention epochs, and $\mu_{R}$ stands for the same in relax (intention absent) epochs. As a consequence of $H_{1}$, all probabilities reported throughout the study are two-tailed.

\subsection{Participants}

Participant recruiting looked for subjects interested in the investigated phenomena and who, based on some regular practice, showed a propensity for absorptive skills. This was motivated by Radin et al's correlation results, and favored meditators, mediums, holistic therapists, psychonauts, artists, martial artists, and athletes. Besides those groups, the recruiting included individuals who, by their curiosity and openness, were highly motivated to take part in the experiment.

The first invitations were sent to a list of experimenter's acquaintances who met the abovementioned group inclusion criteria. Then, some who took part in the experiment were asked to nominate new potential participants from their own acquaintances, thus implementing a snowball sampling. The biased sample presented no obstacle as the main question concerned the existence of the investigated phenomenon, regardless of effect size distortions caused by a supposedly privileged group. In particular, in an experiment where attention is a crucial ingredient, it's convenient to select volunteers who, by their interest and motivation, are more likely to perform the experimental task with an increased level of commitment.

After their selection, the recruited volunteers filled out an online form about their personal practices and their beliefs and experiences regarding anomalous phenomena. The form also included a Portuguese translated version of the Tellegen absorption scale [23]. On the session days, before and after the experimental task, the participants filled out a questionnaire examining their current psychological state. A discussion of the correlations obtained between the questionnaires and scales with the experimental results lies outside the scope of the present work, and will be left for a future publication.

Across the experiment, no tests prior to the planned sessions were performed in order to pre-select the candidates. However, $29 \%$ of the sessions consisted of returning participants re-invited because of their previously obtained high $z$-scores.

\subsection{Double-slit optical system}

The double-slit system geometry is presented in Fig. (3). After traveling $38 \mathrm{~cm}$ the diverging laser beam reaches the double-slit region as a monochromatic plane wave of $\lambda$ wavelength. The wavefront is then diffracted by the two rectangular apertures with respective widths of $s_{1}$ and $s_{2}$, which are separated by a $d$ length. The distance from slit $j$ center to an $x$ point in the camera sensor is given by $r_{j}=\sqrt{y^{2}+x_{j}^{2}}$, where $j=1,2 ; x_{1}=\left(x-x_{0}\right)+\left(s_{1}+d\right) / 2$; $x_{2}=\left(x-x_{0}\right)-\left(s_{2}+d\right) / 2 ;$ and $x_{0}$ is the centrally-symmetric position between both slits.

According to the scalar diffraction theory [24, p. 75], the wavefield strength $U$ at a point $x$ can be expressed as a superposition of spherical waves emanating from every point within the diffraction aperture. The Huygens-Fresnel principle (as predicted by the first RayleighSommerfeld solution) followed by a Fraunhofer approximation results in the following intensity 


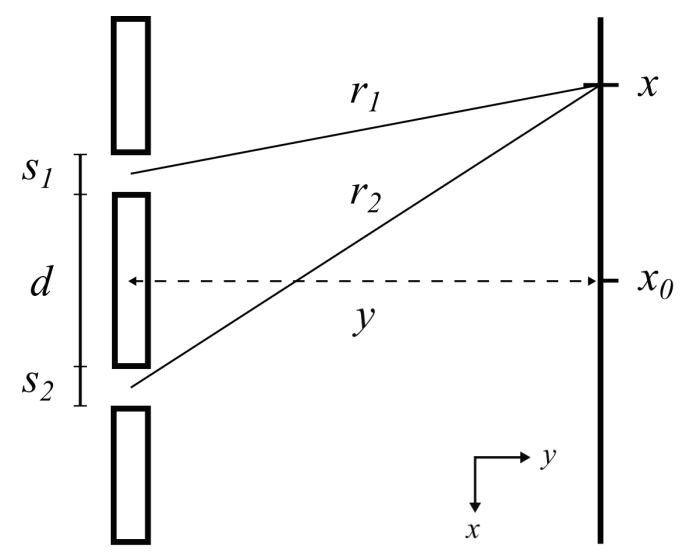

Figure 3: Top view of the double-slit system geometry. The double-slit $x z$ plane is placed at a fixed $y$ distance from the camera sensor $x z$ plane.

after a single slit $j$ :

$$
\begin{array}{r}
U_{j}(x)=U_{j}^{0} \exp \left\{i\left[\theta_{j}+\frac{2 \pi y}{\lambda}+\frac{\pi}{\lambda y} x_{j}^{2}(x)\right]\right\} \frac{\sin \beta_{j}(x)}{\beta_{j}(x)}, \\
\beta_{j}(x)=\frac{\pi}{\lambda y} s_{j} x_{j}(x),
\end{array}
$$

where $U_{j}^{0}$ represents the total field strength emanating from the slit, and the phase $\theta_{j}$ translates a possible small rotation of the slit plane over the $z$ axis. The measured light intensity $I$ in the CCD sensor plane is given by the two-slit field superposition $\left|1 / \sqrt{2} U_{1}+1 / \sqrt{2} U_{2}\right|^{2}$, and in more detail to:

$$
\begin{array}{r}
I(x)=\frac{1}{2} I_{1}(x)+\frac{1}{2} I_{2}(x)+\cos \left\{\frac{\pi}{\lambda y}\left[x_{2}^{2}(x)-x_{1}^{2}(x)\right]+\theta_{r}\right\} \sqrt{I_{1}(x) I_{2}(x)}+D C, \\
I_{j}(x)=U_{j}(x) U_{j}^{*}(x),
\end{array}
$$

where $\theta_{r}=\theta_{2}-\theta_{1}$, and $D C$ represent the dark current noise in the camera CCD. The first and the second terms are the diffraction components, and the third term is the interference component. All components together form an interference pattern, as exemplified in Fig. (21).

A least-square curve fitting procedure using Eq. (3) is applied to extract the physical parameters of the experimental setup. The data sample used consists of 100 CCD frames (equally time-spaced) obtained from each of the 60 control sessions formed by the first experiment. For practical purposes Eq. (3) is rewritten: $\left(U_{1}^{0}\right)^{2}$ is factored out from the three first members and $U_{r}=U_{2}^{0} / U_{1}^{0}$ is introduced in the next two; the $x$ value is converted to a discrete set using the relationship $x=\left(i-i_{0}\right) \Delta p$, where $i=0, \ldots, 1263$ and $\Delta p$ is the pixel size. The extracted parameters are shown in Tab. (1).

\section{7 $\quad$ Model}

A theoretical model is developed to identify the experimental signatures arising from a legitimate mind-matter interaction. The interaction dynamics are modeled by a binary choice $c= \pm 1$, an intensity $0 \leq \psi \leq 1$, and a phase difference $-\pi<\phi \leq \pi$. The three degrees of freedom are considered functions of the conscious agent's subjective state. The extended interference pattern equation accommodating the supposed interaction is then given by:

$$
I(x, c, \psi, \phi)=\left|\sqrt{\frac{1+c \psi}{2}} U_{1}(x)+\sqrt{\frac{1-c \psi}{2}} e^{i \phi} U_{2}(x)\right|^{2}+D C,
$$




\begin{tabular}{|c|ccc|}
\hline par & mean & std & unit \\
\hline$y$ & 30.458 & 0.017 & $\mathrm{~mm}$ \\
$s_{1}$ & 12.56 & 0.11 & $\mu \mathrm{m}$ \\
$s_{2}$ & 12.18 & 0.16 & $\mu \mathrm{m}$ \\
$U_{1}^{0}$ & 157 & 2 & $\mathrm{~mm}$ \\
$D C$ & 374 & 42 & - \\
$U_{r}$ & 0.940 & 0.022 & - \\
$\theta_{r}$ & -0.102 & 0.041 & - \\
\hline$d$ & 200 & - & $\mu \mathrm{m}$ \\
$\lambda$ & 635 & - & $\mathrm{nm}$ \\
$\Delta p$ & 3.75 & - & $\mu \mathrm{m}$ \\
$i_{0}$ & 652 & - & - \\
\hline
\end{tabular}

Table 1: Parameters mean values and standard deviations obtained in the fitting procedure of the 6,000 CCD frames. No std indicates a parameter that was fixed during the fitting procedure.

where global conservation of the light intensity is ensured as the sum of the two coefficients squared norm equals one. In more detail:

$$
\begin{gathered}
I(x, c, \psi, \phi)=\frac{1+c \psi}{2} I_{1}(x)+\frac{1-c \psi}{2} I_{2}(x)+ \\
+\sqrt{1-\psi^{2}} \cos \left\{\frac{\pi}{\lambda y}\left[x_{2}^{2}(x)-x_{1}^{2}(x)\right]+\theta_{r}+\phi\right\} \sqrt{I_{1}(x) I_{2}(x)}+D C .
\end{gathered}
$$

Inspecting Eq. (5) one learns that a $\psi$ action would increase the amount of light diffracted through a specific single slit while decreasing the amount through the other. The binary $c$ choice expresses the specific slit to be light-enhanced: $c=1$ meaning slit 1 and $c=-1$ meaning slit 2. For a non-zero $\psi$, the interference term decreases independently from $c$. A $\phi$ action would shift the interference term to the left/right depending on its sign.

Although it's possible to work with the time-domain pattern, the information extraction using fitting procedures requires intensive computations. Facing this technical challenge, it's convenient to Fourier transform the light intensity (operation denoted as $\mathcal{F}\{I\}$ ) using fast algorithms, and search for interaction signatures in the $k$ frequency domain.

The next question to be addressed concerns the effect of $\psi \phi$-perturbations in the interference pattern and its translation into the magnitude and phase Fourier components. For a given double-slit system geometry, what are the magnitude and phase $k$-values that are more sensitive to the investigated $\psi \phi$-influences? The answer to this question will lead to the most efficient strategy for probing the interaction existence. The following component differences are adopted as a metric to characterize the signal associated with $\psi \phi$-perturbations:

$$
\Delta M(k)=M_{\mathrm{int}}(k)-M_{\mathrm{rlx}}(k) \quad ; \quad \Delta P(k)=\frac{P_{\mathrm{int}}(k)-P_{\mathrm{rlx}}(k)}{M_{\mathrm{rlx}}(k)},
$$

where $\mathcal{F}\{I(x, c, \psi, \phi)\}=M_{\text {int }} \exp \left(i P_{\text {int }}\right)$ represents the $\psi \phi$-influenced information supposedly obtained in intention conditions, and $\mathcal{F}\{I(x, 0,0,0)\}=M_{\text {rlx }} \exp \left(i P_{\text {rlx }}\right)$ represents the $\psi \phi$-absent information from relax conditions. The phase difference is divided by the associated magnitude to correctly represent the variation inertia - it's easier to change the phase of smaller magnitude $k$-values. Using the fit-extracted physical parameters from Tab. (11) and Eqs. [5, 6, the $\psi \phi$ interacting versus non-interacting differences are numerically evaluated and presented in Fig. (4).

The difference extrema are used to guide the construction of variables of interest sensitive to $\psi \phi$-action. For example, a variable can be designed as the area of the magnitude component 

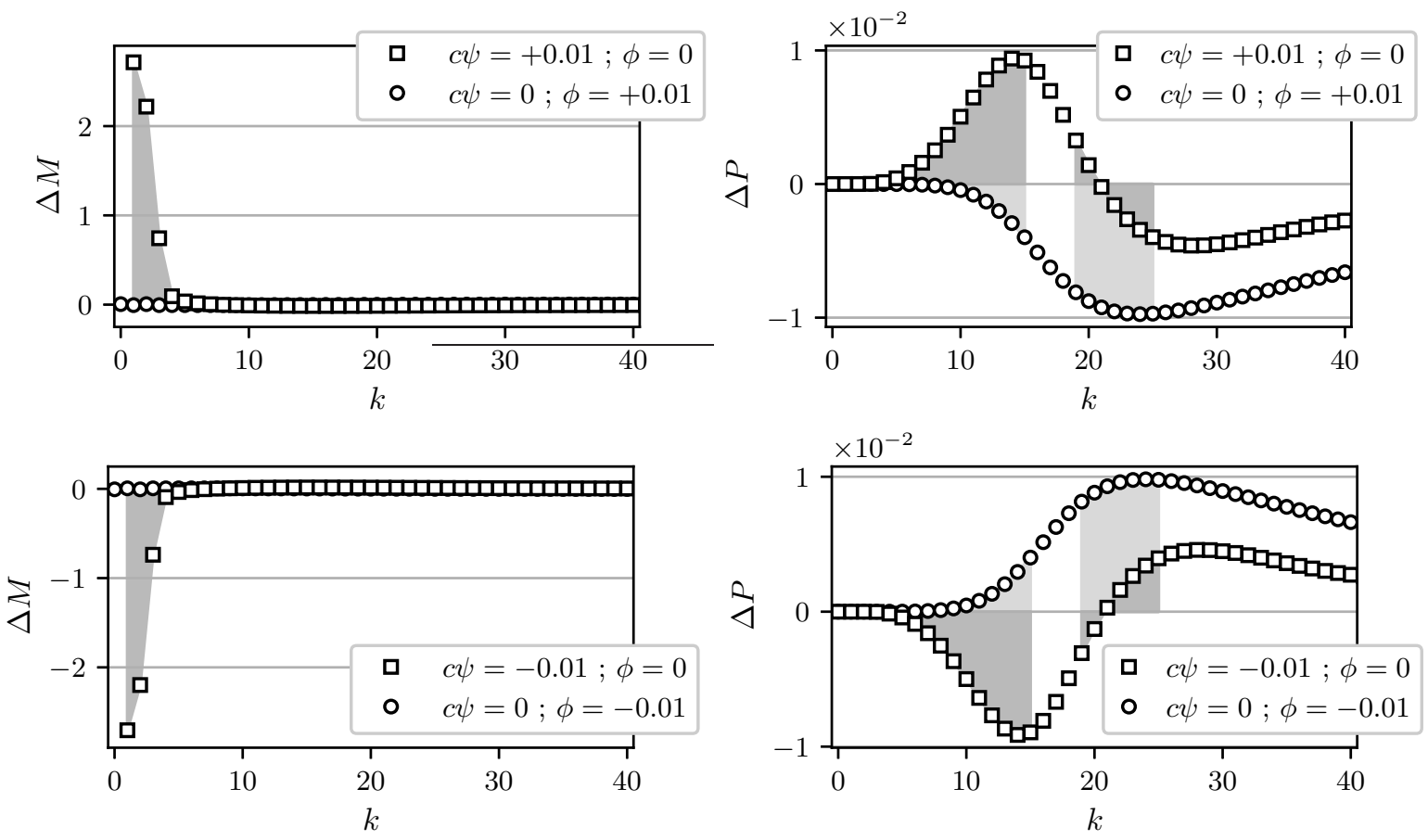

Figure 4: Magnitude and phase difference signatures for different values of $c, \psi$ and $\phi$. The magnitude shaded region represents the area explored in the real-time feedback, while the phase shaded areas represent the two chosen $k$-windows for building the $V_{1}$ and $V_{2}$ variables of interest used in the analysis. The differences sign inversion resulting from a $c$ sign inversion allows one to discriminate between the increase or decrease in the light intensity diffracted through a specific slit.

evaluated between the $k=1 \ldots 4$ window. In this way, a CCD frame is translated into a single real number that should increase when the first slit diffraction intensity increases, or decrease when the second slit diffraction intensity increases. The standard score sign obtained by a statistical test comparing the mean of the variables obtained in intention and relax conditions will then reveal the enhanced single-slit. It's important to note that the discrimination between $c=+1$ and $c=-1$ depends on $I_{1}(x)$ and $I_{2}(x)$ having slightly different shapes. Conversely, the phase component does not depend on geometrical asymmetries in the slits to allow a discrimination of the enhanced single-slit. Those arguments are demonstrated in the Supporting Information (SI) Section Appendix S1.

While the magnitude is insensitive to $\phi$ variations, the phase difference reveals a first $k<17$ region dominated by a $c \psi$ action and a second $k>17$ region dominated by $\phi$ contributions. Those two phase regions are represented throughout this study by variables respectively denoted as $V_{1}$ and $V_{2}$.

\subsection{Real-time feedback}

The feedback system is designed to inform the participant about instantaneous variations in the amount of light crossing through a specific single-slit. This is accomplished by obtaining this information on the fly, and then transforming it into a feedback magnitude as a real number ranging from 0 to 1 used to modulate the feedback devices intensity. While a feedback magnitude of 0.5 means that no variation is taking place, a value between 0.5 and 1 means an increase in the amount of light passing through the target slit, and a value between 0 and 0.5 means a decrease in the same quantity.

The participant is in sensory contact with two feedback devices: noise-canceling headphones 
playing a richly harmonic droning tone and the colorful light produced by a LED shining through translucent glass. As the feedback magnitude increases, the LED light shines more brightly in the dark experimental room, and is accompanied by a corresponding increase in the tone volume. The feedback light colors are randomly picked for each of the 20 intention runs from a pool of 8 different pre-defined colors. During the data collection, the following method is used to calculate and inform the feedback magnitude. At every frame:

1. A fast Fourier transform is applied to the CCD frame, and the magnitude component is used to calculate the experiment-specific feedback variable of interest. The variables definitions are presented in Section 3.2 .

2. Two sliding window vectors are updated with the variable value, storing respectively the last 30 and 150 frame values.

3. A Mann-Whitney $\mathrm{U}$ test is applied to the two samples. The resulting $z$-score is used to calculate a one-tailed probability $p$. The hypothesis being tested (fixed for each experiment) is interpreted as the last 3 -second variable mean being significantly greater, or less, than the last 15 -second variable mean.

4. The feedback magnitude is obtained as $F=1-p$, and $F$ is set to a minimal value of 0.1 if below this threshold.

5. To avoid sudden changes that may disrupt the participant's concentration, the mean of the last $20 F$ values is calculated to $F_{m}$.

6. If the current frame is associated with an intention condition, the $F_{m}$ value is used to instantly modulate the light and volume intensity of the feedback devices. Alternately, if the experiment is in the relax condition, no information about the experiment state is given to the participant; the feedback light remains off, and the sound is kept at a fixed 0.3 intensity. As a result, the feedback light is turned on only during intention runs, while the feedback volume is kept on during the whole session, being only modulated during intention runs and kept at a fixed 0.3 intensity during relax and during the recorded conditions announcements.

The feedback mechanism simplifies the task description, serving as an interface between the conscious agent and the physical process dynamics. Without it, the task instructions might sound rather abstract, causing mental wandering and distractions during the experiment. To simplify, the participants are instructed to always intend the increase of the feedback magnitude during the intention runs. The information about the favored slit is kept blind to the participant, but they are informed that a magnitude increase is linked to a physical variation, so by focusing on the feedback, they are indirectly interacting (or attempting to interact) with the light crossing the apparatus. As a secondary role, the feedback is used to arouse the participants' motivation in the hopes that they will eventually experience some sort of correlation between the presented intensities and their subjective state, thereby reinforcing their attention and intention toward the experimental system.

\subsection{Analysis}

The CCD frames recorded during each experimental session are processed and transformed into variables of interest according to the following steps (see Section 2.2 for the variables definitions):

1. For every $n$ frame, the CCD frame array $I[n, i]$ is transformed by a fast Fourier algorithm, and decomposed into magnitude $M[n, k]$ and phase $P[n, k]$ polar components, where $k=$ $0,1, \ldots, 631$. 
2. For every $k$, the phase is unwrapped along the $n$ frames to $P_{u}[n, k]$ in order to remove misleading $2 \pi$ discontinuities caused by the $-\pi$ to $\pi$ constraint.

3. For every $k$, the standard deviation of $P_{u}[n, k]$ along the $n$ frames is computed to $s_{P}[k]$.

4. A variable of interest is obtained as $V_{\alpha}[n]=\sum_{k=l}^{h} P_{u}[n, k]$, for every $k$ between $l$ and $h$ that satisfies the $s_{P}[k]<0.5$ relationship. In the case of a non-satisfying condition, the given $k$ is left out of the sum, not contributing to the variable in that particular session - this cut is described in more detail in SI Section Appendix S2. The $V_{\alpha}$ variable is optionally followed by the notation $\langle l-h\rangle$ to specify its lower and higher $k$-window bounds.

5. Additionally, compound variables can be obtained as e.g. $V_{12}[n]=+V_{1}[n]\langle 5-15\rangle-$ $V_{2}[n]\langle 19-25\rangle$.

Using a procedure referred to as differential analysis, a nonparametric bootstrap test is applied to the variables of interest in order to test the equality hypothesis between the intention and relax sample means. The difference of the intention and relax variable's mean values is initially computed using the original conditions array. Then, the test repeatedly shifts the condition array to a random frame position in order to find the statistical distribution of the intention/relax differences. For each session and variable, a standard $z$-score is obtained with the following steps:

6. An $8^{\text {th }}$ order polynomial is least-square fitted to the variable $V[n]$. The residual difference between the variable and the polynomial is obtained as $V_{d}[n]$. This nonlinear detrending procedure is made in order to rule out the variable dependency in slowly changing environmental conditions, e.g. room temperature.

7. The run array $R[n]$ is used to identify the first frame $n_{s}$ of the first attention run, as well as the last frame $n_{e}$ of the last relax run. To avoid artifacts in the variable extremities caused by the polynomial fitting procedure, the variable $V_{d}$ is trimmed in the range $n_{s}-300$ to $n_{e}+300$, being then denoted as $V_{d}\left[n_{t}\right]$, where $n_{t}=0,1, \ldots, n_{e}-n_{s}+600$.

8. The condition array $C[n]$ is trimmed in the same interval (described in the previous item) to $C\left[n_{t}\right]$, and then used to split the variable $V_{d}\left[n_{t}\right]$ into two arrays: $V_{I}[m]$ and $V_{R}[m]$ with $m=0,1, \ldots, 5999$ values respectively recorded during intention and relax conditions.

9. $V_{I}[m]$ and $V_{R}[m]$ means are calculated to $\mu_{I}$ and $\mu_{R}$. The two-sample mean difference is denoted as $\Delta \mu=\mu_{I}-\mu_{R}$. The null hypothesis is $\mu_{I}=\mu_{R}$, while $\mu_{I} \neq \mu_{R}$ stands for the alternative hypothesis.

10. A pseudorandom number $r$ between 0 and $n_{t}$ length is drawn using a Mersenne Twister algorithm. $C\left[n_{t}\right]$ is copied and circularly shifted by $r$ units, resulting in $C_{r}\left[n_{t}\right]=C\left[n_{t}-r\right]$. The procedure described in items 8 and 9 is then applied to $C_{r}$, resulting in the mean difference $\Delta \mu_{r}$.

11. The previous item procedure is repeated 5,000 times, filling a vector with $\overline{\Delta \mu_{r}}$ mean and $\sigma_{\mu r}$ standard deviation.

12. The standard score concerning the intention-relax sample mean difference is obtained as $z=\left(\Delta \mu-\overline{\Delta \mu_{r}}\right) / \sigma_{\mu r}$.

Figure 5 presents an example of a $V_{12}$ compound variable obtained in a participant session. The top plot shows the variable (black line) obtained by following steps 10 5, and the associated best fitting $8^{\text {th }}$ order polynomial (white line). The bottom plot displays the variable residual (black line) as described in step 6, and (for illustrative purposes) the residual average obtained 
through a 300-frame window Savitzky-Golay filter (white line). Both data samples are trimmed as described in step 7, and show the condition data described in step 8- dark gray bars represent intention and light gray bars represent relax condition frames.
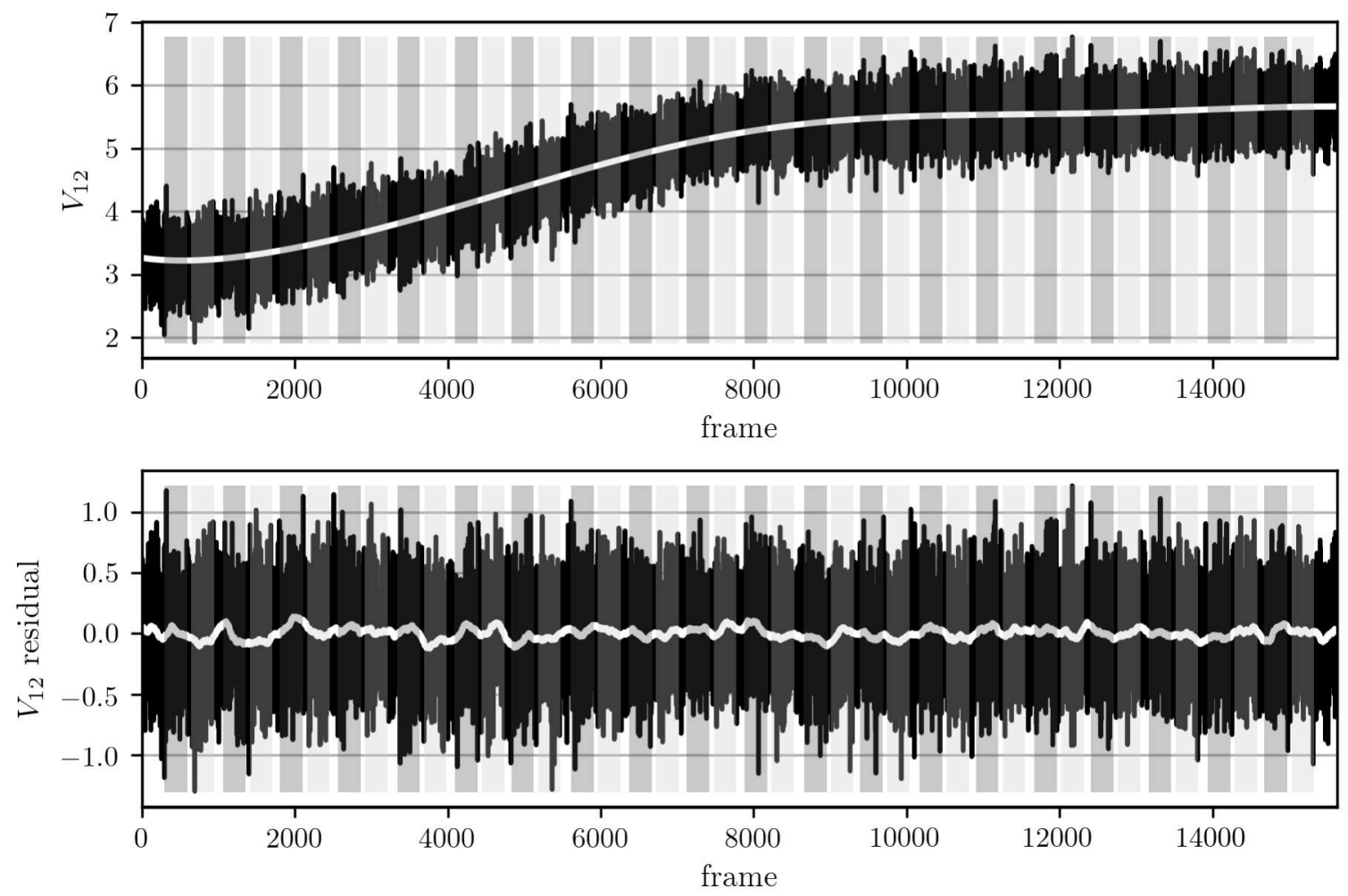

Figure 5: Variable of interest example. This particular session composed $V_{12}$ as $V_{1}\langle 5-15\rangle-$ $V_{2}\langle 19-25\rangle$, and resulted in a $z=1.65$ score for this variable.

For an experiment consisting of $N$ sessions, a global $z$-score for a given variable is obtained by combining individual session results in a Stouffer's $z=\sum_{i=1}^{N} z_{i} / \sqrt{N}$. The effect size is then calculated by $e s=z / \sqrt{N}$, with $\sigma=1 / \sqrt{N}$ standard error.

\section{Results}

\subsection{Study design}

Compared with previous efforts to probe the phenomenon using random number generators (RNG), the double-slit (DS) system has the advantage of providing interference information across a spatial dimension rather than providing binary outcomes. Having more information available makes it more potentially sensitive to the investigated $\psi \phi$-interaction. However, the richness comes at the cost of requiring a more complex analysis to extract the relevant information.

While in an RNG experiment the null hypothesis is precisely defined as the $0 / 1$ data conforming to the associated binomial distributions, the solution in a DS experiment is far more complex. It starts with two questions: what is the variable of interest most sensitive to the investigated interaction, and what is the most appropriate statistical test to evaluate the differences between the intention and relax conditions? Concerning the use of Fourier-transformed variables, it's not possible to simply mirror the definitions used in a different experimental arrangement as, by numerical inspection, one finds that the meaningful model predicted $\psi \phi$ $k$-windows are sensitive to small variations in the geometry parameters such as the double-slit distance from the camera sensor. 
Adding to the complexity, the investigated interaction is supposed to display a goal-oriented aspect according to the reported RNG meta-analyses literature. This means that the degrees of freedom $c, \psi$ and $\phi$ should vary in a specific way to fulfill the participant intention of a feedback magnitude increase. As a consequence of this plasticity, the collected data should itself depend on the provided feedback characteristics, and stronger results may be obtained by providing more reliable real-time information about the slits intensity variations. Conversely, providing meaningless feedback could render participant data statistically equivalent to the controls. At early stages, one finds a paradoxical situation: sensitive information should be presented to the participants at the same time that a posterior data analysis (with enough statistical power) is required in order to define the relevant variables.

Facing those challenges, it's clear that starting a DS experiment with a pre-defined analysis is a good recipe for obtaining non-significant differences between the intention and relax conditions, or, a false negative result if the investigated effect is genuine. Thus, for every novel setup, it's necessary to start the experiment in an exploratory fashion. At the same time, the more the researcher explores the analysis degrees of freedom, the more likely this will create falsepositive results. To balance this delicate equation, the final analysis variables used in this study are obtained by an optimizing procedure applied to partial data. The optimized analysis is then uniformly applied to the remaining datasets. Two optimization scenarios are considered: the variables that maximize the intention/relax differences in the participant, and those in the control data. Finally, to investigate whether the results can be explained by over-tuning, additional experiments are proposed with a pre-planned analysis using the same optimized method.

For completeness' sake, it's important to state that the theoretical model presented in Section 2.7 was not available prior to the data collection. The variables initially used were based on the magnitude component of the Fourier transform, and were analyzed using a different method than the one presented in Section 2.9. After finishing the experiments 1-5 data collection, the analysis method was improved by the introduction of polynomial non-linear detrending - a more meaningful approach than the previously used linear detrending. However, a global analysis applying the new method to the same magnitude variables turned into a non-significant result. This led the experimenter to consult the digital signal processing literature, finding that the initial estimate of magnitude-based variables wasn't optimally effective since "much of the information about the shape of the time domain waveform is contained in the phase, rather than the magnitude" [25, p. 192]. The Fourier magnitude component is more appropriately used when dealing with an oscillating variable collected across time, while for the spatially-distributed interference pattern, the waveform shape is more relevant than its particular frequency spectrum. This understanding guided the model development, and led to the use of phase variables. As this finding occurred after the $N=160$ series, the experimenter didn't have the opportunity to test the feedback with the same phase variables used in the final analysis.

For the exploratory experiments, 127 volunteers contributed 160 experimental sessions resulting in five experimental blocks labeled 1 to 5 , with each having a pre-planned number of participants. The data collection followed the procedure described in Section 2.3, and occurred over a timespan of 9 months starting in October 2016. Data for experiments 4 and 5 were collected during a 40-session block that alternated each day between exp. 4 and exp. 5 .

In an experiment labeled 0,30 sessions were recorded following the same procedure used in experiments 1-5, the only difference being that a $150 \mathrm{~W}$ lamp replaced a person during the participant sessions. The lamp was placed in the participant chair inside a black cardboard cylinder, and was turned off before the control session started.

In the formal experiments labeled 6-9, 44 new volunteers, plus 26 that took part in the previous experiments contributed 80 experimental sessions. The analysis methodology preregistered in [26,27] was the same used in the exploratory experiments. Data for experiments 6 and 7 were collected during a 40-session block that alternated each day between exp. 6 and exp. 
7. The same occurred for experiments 8 and 9 . The two experimental blocks were separated by a three week period. The $N=80$ data collection occurred over a timespan of 2 months starting in October 2017.

\subsection{Feedback configuration}

As described in Section 2.8, the feedback configuration consists of two experimenter choices: a variable of interest and a binary single-tailed test hypothesis. By fixing these choices the experimenter defines the binary $c$, while the participant (blind to the $c$ definition) accounts for the $\psi \phi$-action.

In experiment 5 , the feedback variable was built using the log-transformed $M$ magnitude component of the Fourier transform, and defined as the area across the $k=1 \ldots 4$ range. The feedback hypothesis (represented by the $>$ symbol) tested an increase of the variable's mean in the short $3 \mathrm{~s}$ window, as compared to the $15 \mathrm{~s}$ one. The larger the variable mean increase, the lower the $p$ probability obtained from the single-tailed test, hence the larger the $F$. The instantaneous $F$ increase in this experiment is expected to reflect a positive $c \psi$ effect, as revealed in Fig. (4), i.e. an increase in the diffraction power through slit 1 . In experiment 4 , the feedback was configured with the same variable but the opposite $<$ test hypothesis. In this case, an $F$ increase is related to an increase in the number of photons crossing the second slit.

The definitions used in each experiment are shown in Tab. (2). The use of different feedback variables in experiments $0-5$ reflects the learning curve of the author as the study evolved. Although different variables have been explored, they all use the first two to five magnitude $k$ wavenumbers, the most sensitive magnitude region for a supposed $\psi$ action. The first experiment used a ratio mathematically defined as $\sum_{k=49}^{55} M[n, k] / \sum_{k=1}^{4} M[n, k]$, and represented as $M$ $\langle 49-55\rangle /\langle 1-4\rangle$. The nominators used in experiments $0-3$ have much smaller predicted variations as compared with the denominators. Thus, the denominators dominate the variable change in the case of a $\psi$ action, implying an inversion in the first three experiments between the test hypothesis and the enhanced slit: an increase in slit 1 (2) diffraction power with the feedback $<(>)$ hypothesis.

\begin{tabular}{|c|c|c|c|}
\hline exp. & FV & FH & FS \\
\hline 0 & $M\langle 49-55\rangle /\langle 1-4\rangle$ & $<$ & 1 \\
1 & $M\langle 49-55\rangle /\langle 1-4\rangle$ & $<$ & 1 \\
2 & $M\langle 5-9\rangle /\langle 1-5\rangle$ & $>$ & 2 \\
2 & $P\langle 5-9\rangle /\langle 1-5\rangle$ & $>$ & 1 \\
3 & $M\langle 3-10\rangle /\langle 1-2\rangle$ & $>$ & 2 \\
4 & $\log M\langle 1-4\rangle$ & $<$ & 2 \\
5 & $\log M\langle 1-4\rangle$ & $>$ & 1 \\
\hline 6 & $\log M\langle 1-4\rangle$ & $<$ & 2 \\
7 & $\log M\langle 1-4\rangle$ & $>$ & 1 \\
8 & $\log M\langle 1-4\rangle$ & $<$ & 2 \\
9 & $\log M\langle 1-4\rangle$ & $>$ & 1 \\
\hline
\end{tabular}

Table 2: Feedback configuration. Variable of interest (FV) and one-tailed test hypothesis (FH), followed by the feedback-favored slit (FS).

Concerning the evolution of the feedback variables, in experiment 1 the variable nominator was associated with the magnitude peak seen in Fig. (2). This focus on the waviness region initially investigated a possible state reduction induced by the observers. In this case a decrease was expected as a consequence of the increase in particle-like photons. Afterwards, the developed model revealed a predicted small $\sqrt{1-\psi^{2}}$ variation linked to the slits intensity mod- 
ulation. In experiment 2 , an extra feedback variable with the same magnitude $k$-window was added using the phase component. Examining the phase difference in Fig. (44), it's possible to see that the nominator dominates in this case, indicating that the diffraction favored slit 1 , while the magnitude variable favored slit 2 . The $z$-score resulting from the phase and the magnitude variables variation test were combined to calculate the feedback magnitude, leading to a contradictory slit enhancement. In contrast, the phase variable unequivocally favored a negative $\phi$ action and an increase in $V_{2}$.

The specific combination of the feedback variable and the feedback hypothesis implies in a feedback-favored slit for each experiment. As shown in Section 2.7, switching from $c=+1$ to $c=-1$ causes a differential sign inversion in the $V_{1}$ variable. As a consequence (in a genuine $\psi \phi$ interaction scenario), the $V_{1}$ differential analysis is expected to result in opposite $z$-score signs when applied to experiments with opposite feedback-favored slits. Thus, an analysis composition rule is pre-defined: when combining the $V_{1} z$-scores from different experiments within a Stouffer's sum, the $z$ signs must be inverted between experiments with opposite feedback-favored slits.

For the variable $V_{2}$, no particular differential sign was intentionally favored in the experiments, as the magnitude variables used in the feedback are insensitive to $\phi$ variations. The analysis composition rule, in this case, could not be pre-defined. The exception is exp. 2, where a positive $z$ was favored by the feedback system.

\subsection{Variables' optimization}

The variables of interest used in the analysis are built using the Fourier transform phase component. The differences shown in Fig. (44) are a good starting point for understanding the regions in the frequency domain sensitive to each investigated degree of freedom $\psi$ and $\phi$, however to effectively define the $k$-windows which maximize the supposed signal-over-noise relationship, an optimization technique was devised according to the following rules:

1. The 60 participant and 60 control sessions from experiment 1 are used to obtain the optimal $k$-window parameters that define the variables of interest. Those datasets are exclusively used for optimization purposes, not contributing to the final result analysis.

2. Two variables are prospected, $V_{1}$ and $V_{2}$, corresponding respectively to the $\psi$ and $\phi$ degrees of freedom. Each variable is defined by a phase sum over a $k$-window with $l$ lower and $h$ higher bounds. The notation $V\langle l-h\rangle$ is adopted to express the variables $k$-range.

3. Diverse combinations of $l$ and $h$ values are explored; the procedure described in Section 2.9 is used to obtain a $V\langle l-h\rangle$ variable, and test it within a differential analysis. Therefore, each $(l, h)$ pair yields a $N=60$ global $z$-score representing the differences in variables between the intention and relax conditions. This procedure is equally applied in the participant and in the control data, resulting in two $z$-score two-dimensional surfaces.

4. The variables explored satisfy the condition of being composed of 5 or more $k$-values. This requirement ensures more restrictive variables, as the larger the window length, the less likely it is to produce same-direction phase variations by pure chance. If the phase variations in the $k$-window are a product of noise, they should be composed of positive and negative variations that cancel out when summed.

5. A comparison test is used to find the $(l, h)$ parameters associated with extreme values in the $z$-score surfaces. Two scenarios are considered, the $V_{1,2}$ variables that maximize the $z$-score absolute value in the participant data, and the $\Lambda_{1,2}$ variables associated with the extreme $z$-scores in the control data. These scenarios are respectively referred to throughout the study as default and reverse. 
Figure 6 shows the $z$-score surfaces resulting from the optimization procedure. The optimal variables found for the default scenario are $V_{1}\langle 5-15\rangle$ and $V_{2}\langle 19-25\rangle$, while the variables that optimize the reverse scenario are $\Lambda_{1}\langle 7-18\rangle$ and $\Lambda_{2}\langle 21-36\rangle$.

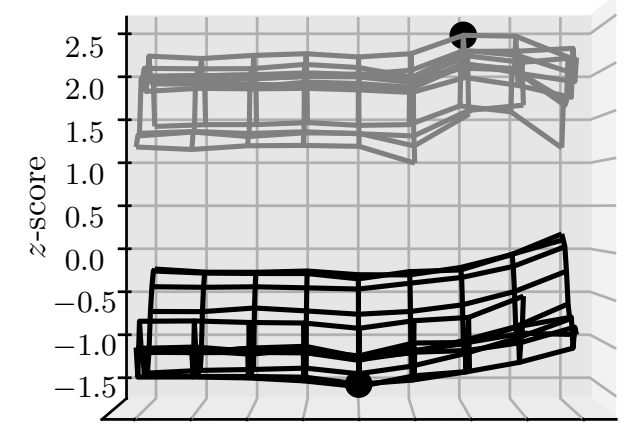

his
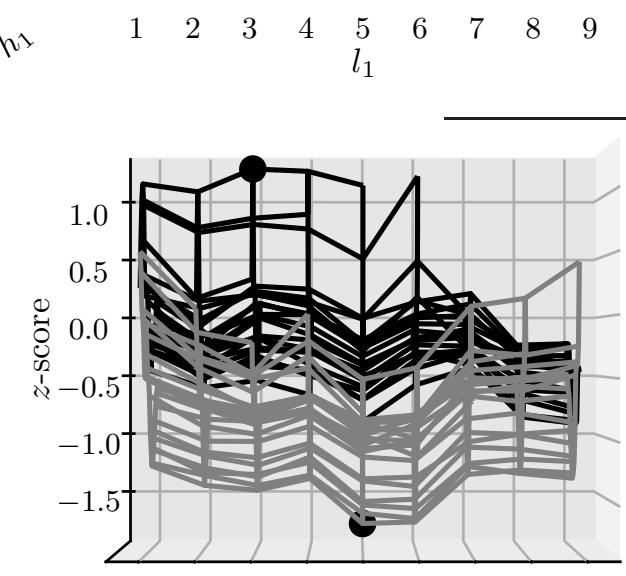

12

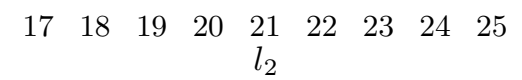

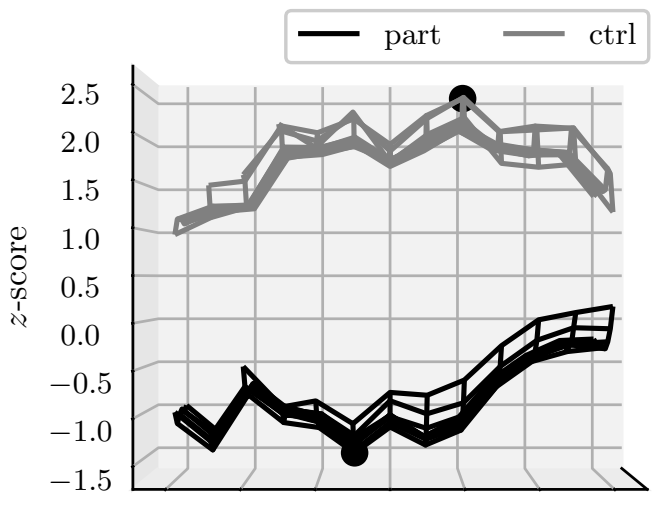

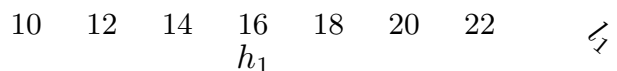

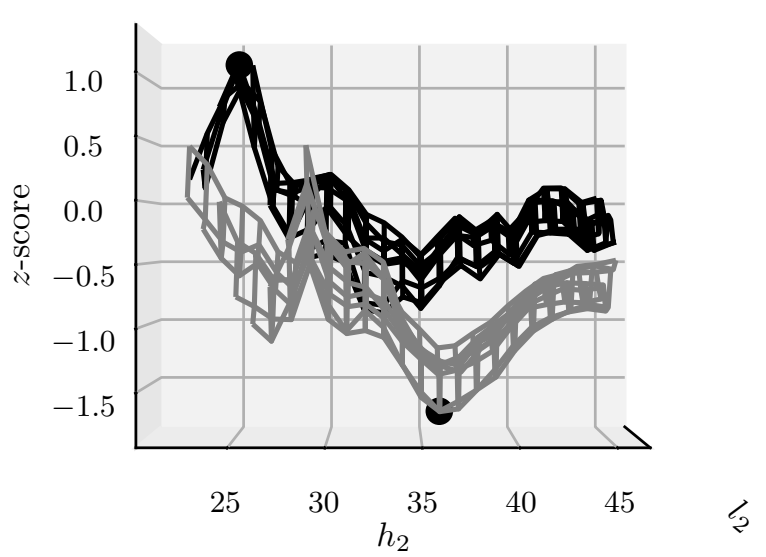

Figure 6: Variables' optimization results. Top: $l$ and $h$ projections of the same participant and control $z$-score surfaces obtained for prospecting $V_{1}$ and $\Lambda_{1}$. Bottom: the same for prospecting $V_{2}$ and $\Lambda_{2}$. Black dots mark the extreme values found for each surface.

\subsection{Exploratory experiments}

The next step consists of uniformly applying the intention/relax differential procedure described in Section 2.9 to the experiments $2-5$ data. Table 3 summarizes the statistical results obtained in the default scenario. Based on the pre-defined composition rule discussed in Section 3.2, an exp. 2-5 Stouffer $z$-score is obtained for $V_{1}$ by reversing the $z$-sign between exps. 2-4 and exp. 5, revealing a significant 3.43 sigma result for the participant data, and $z=0.49$ for the controls. For $V_{2}$, an exp. 2-5 global $z$-score is obtained by reversing the exps. $3-5 z$ sign, revealing a significant 2.80 sigma result for the participant data, and $z=-0.20$ for the controls.

Compound variables $V_{12}$ are obtained by combining $V_{1}$ and $V_{2}$ in a constructive way while respecting the sign composition rules. For experiments 4 and 5 , for example, the composition rules require opposite signs for $V_{1}$ and same negative signs for $V_{2}$. The compound variable is then obtained using a final-score positive sign convention, such that $V_{12}$ for experiments 4 and 5 is respectively obtained as $+V_{1}-V 2$ and $-V_{1}-V 2$ (equally applied in participant and control sessions). The composition is applied to the variable's data before the differential analysis. This signal-amplifying technique benefits from correlated (or anti-correlated) variations in regions 1 and 2. The cumulative $z$-score plots for $V_{12}$ presented in Fig. (7) reveal that the participant 


\begin{tabular}{|cc|cc|cc|ccccc|}
\hline & & \multicolumn{2}{|c|}{$V_{1}\langle 5-15\rangle$} & \multicolumn{6}{|c|}{$V_{2}\langle 19-25\rangle$} & \multicolumn{6}{|c|}{$V_{12}$} \\
\cline { 3 - 10 } exp. & $\mathrm{N}$ & $z_{p}$ & $z_{c}$ & $z_{p}$ & $z_{c}$ & comp. & $z_{p}$ & $z_{c}$ & $e s_{p}$ & $e s_{c}$ \\
\hline 0 & 30 & 0.54 & -0.55 & 0.79 & 0.97 & $+V_{1}+V_{2}$ & 0.85 & 0.21 & 0.15 & 0.04 \\
1 & 60 & -1.49 & 2.00 & 1.16 & -0.58 & $-V_{1}+V_{2}$ & 1.97 & -1.25 & 0.25 & -0.16 \\
2 & 30 & 0.52 & 0.70 & 1.10 & -0.33 & $+V_{1}+V_{2}$ & 1.65 & 0.48 & 0.30 & 0.09 \\
3 & 30 & 1.65 & -0.15 & -0.59 & 0.10 & $+V_{1}-V_{2}$ & 2.07 & -0.39 & 0.38 & -0.07 \\
4 & 20 & 1.85 & -0.13 & -1.32 & 0.87 & $+V_{1}-V_{2}$ & 2.40 & -0.76 & 0.54 & -0.17 \\
5 & 20 & -3.15 & -0.54 & -2.87 & -0.96 & $-V_{1}-V_{2}$ & 3.50 & 1.44 & 0.78 & 0.32 \\
$2-5$ & 100 & 3.43 & 0.49 & 2.80 & -0.20 & & 4.68 & 0.35 & 0.47 & 0.04 \\
\hline
\end{tabular}

Table 3: Exploratory experiments' results in the default scenario. The participant (control) sessions Stouffer's $z$-score is denoted as $z_{p}\left(z_{c}\right)$, while effect size is denoted as $e s_{p}\left(e s_{c}\right)$.

effects are consistently obtained in a crescent fashion across the experimental sessions, rather than being caused by a few deviating sessions. The controls, in turn, show a $z=0$ tendency.

Table 4 shows the statistical results obtained in the reverse scenario. In this variable's scenario, the exercise also consists of implementing sign-composition choices that maximize the control data $z$-scores. The signs used are shown in the comp. column of the results' table. Unlike with the standard scenario $V_{1}$ compositions, the reverse signs are not bound to any physical reasoning. An examination of the $\Lambda_{1}$ variable reveals that the significant $z_{c}$ value obtained in the exp. 1 optimization is not consistently replicated in the remaining experiments. While the $\Lambda_{2}$ variable obtains a slightly significant 2.18 sigma global result in the controls, the $\Lambda_{12}$ composition conforms to the null hypothesis.

\begin{tabular}{|cc|cc|cc|ccccc|}
\hline & & \multicolumn{2}{|c|}{$\Lambda_{1}\langle 7-18\rangle$} & \multicolumn{2}{|c|}{$\Lambda_{2}\langle 21-36\rangle$} & \multicolumn{5}{|c|}{$\Lambda_{12}$} \\
\cline { 3 - 10 } exp. & $\mathrm{N}$ & $z_{p}$ & $z_{c}$ & $z_{p}$ & $z_{c}$ & comp. & $z_{p}$ & $z_{c}$ & $e s_{p}$ & $e s_{c}$ \\
\hline 0 & 30 & 0.85 & -0.43 & 1.01 & 0.66 & $-\Lambda_{1}+\Lambda_{2}$ & 0.40 & 0.67 & 0.07 & 0.12 \\
1 & 60 & -1.05 & 2.45 & -0.68 & -1.73 & $+\Lambda_{1}-\Lambda_{2}$ & -0.32 & 2.55 & -0.04 & 0.33 \\
2 & 30 & 0.93 & -0.83 & -1.08 & -0.72 & $-\Lambda_{1}-\Lambda_{2}$ & -0.04 & 0.47 & -0.01 & 0.08 \\
3 & 30 & 0.46 & -0.32 & -1.79 & 1.36 & $-\Lambda_{1}+\Lambda_{2}$ & -1.92 & 1.29 & -0.35 & 0.24 \\
4 & 20 & 1.54 & -0.48 & -0.51 & 1.04 & $-\Lambda_{1}+\Lambda_{2}$ & -1.39 & 0.25 & -0.31 & 0.06 \\
5 & 20 & -0.56 & -0.88 & -0.55 & -1.29 & $-\Lambda_{1}-\Lambda_{2}$ & 0.02 & 1.70 & 0.00 & 0.38 \\
$2-5$ & 100 & -1.20 & 1.24 & -0.37 & 2.18 & & -1.68 & 1.84 & -0.17 & 0.18 \\
\hline
\end{tabular}

Table 4: Exploratory experiments' results in the reverse scenario. The participant (control) sessions Stouffer's $z$-score is denoted as $z_{p}\left(z_{c}\right)$, while effect size is denoted as $e s_{p}\left(e s_{c}\right)$.

The $V_{12}$ effect sizes throughout the experiments can be seen in Fig. (8). The results for the standard scenario show statistically significant deviations in the participant sessions, and null effects in the controls. The reverse exercise fails to produce a globally significant result in the control sessions. Furthermore, it produces similar effect size magnitudes for the control and the participant data. The inability to obtain an artificial significant result in the control data by exploiting the optimization method and the variable composition provides evidence for a legitimate interaction in the participant data.

In experiment 0 , the chosen signs for the compound variable $V_{12}$ maximize $z_{p}$ in an attempt to artificially produce a significant result. The null effect results obtained in this experiment dismiss a room-temperature increase as an evident artifact that could explain the participant results in exps. $1-5$.

To study the $\psi \phi$-interaction homogeneity along the sessions duration, the variables' residuals and the condition arrays were divided in half before the differential analysis. In experiments 
Exp. 0

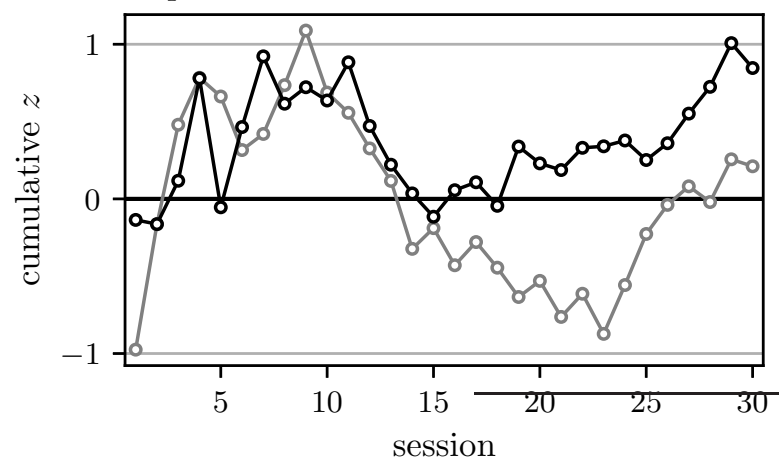

Exp. 2

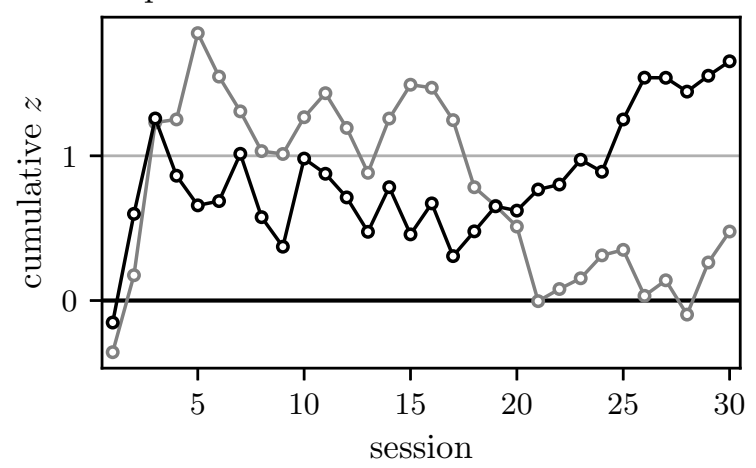

Exp. 4

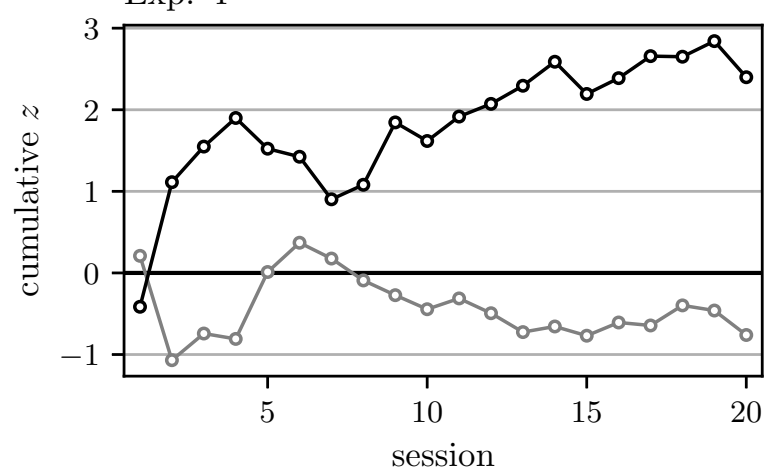

Exps. 2-5

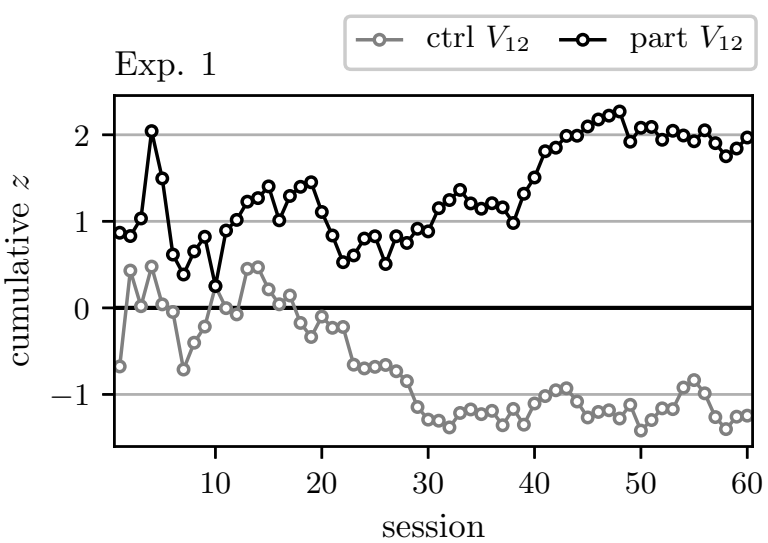

Exp. 3

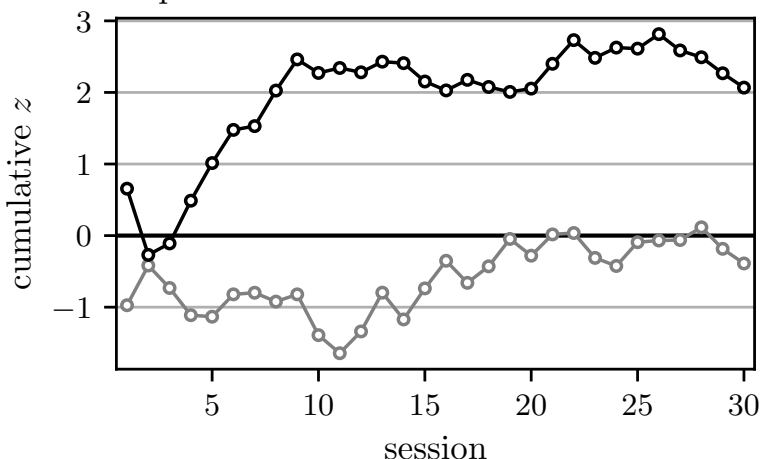

Exp. 5

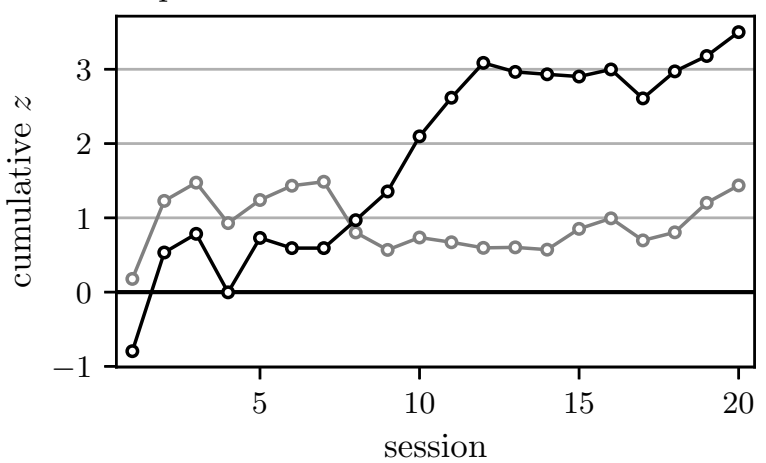

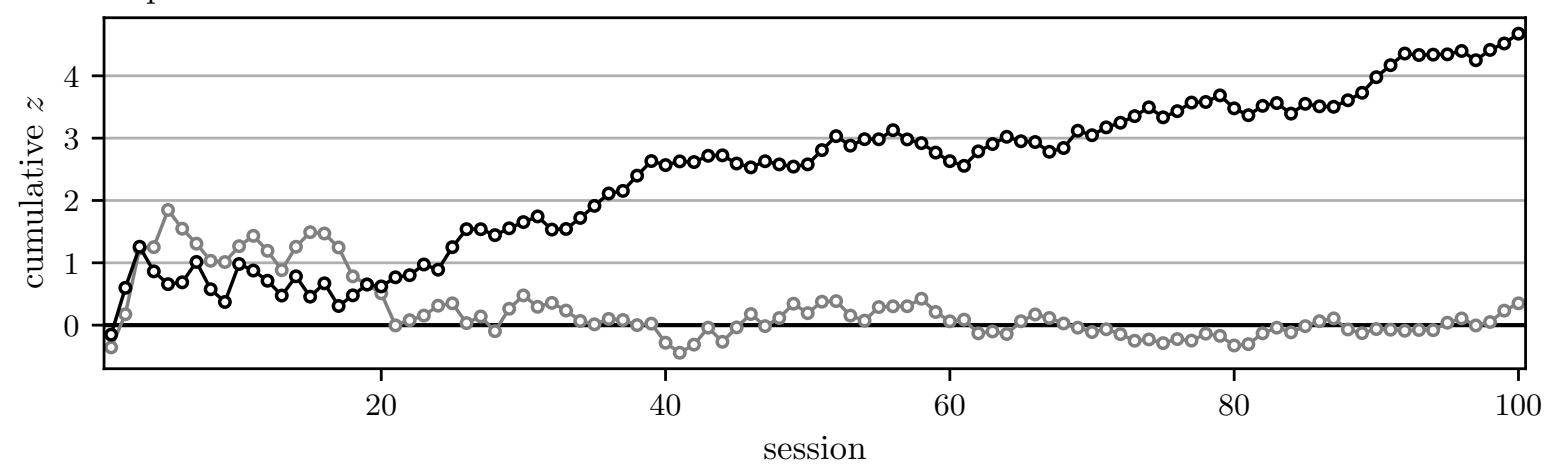

Figure 7: Cumulative z-score for the exploratory experiments in the default scenario. Cumulative plots are calculated as $\sum_{i=1}^{s} z_{i} / \sqrt{s}$, and given as a function of the session number $s$. The last points represent the values shown in Tab. (3). The bottommost plot reveals the Stouffer global result for the 100 sessions from experiments $2-5$. 


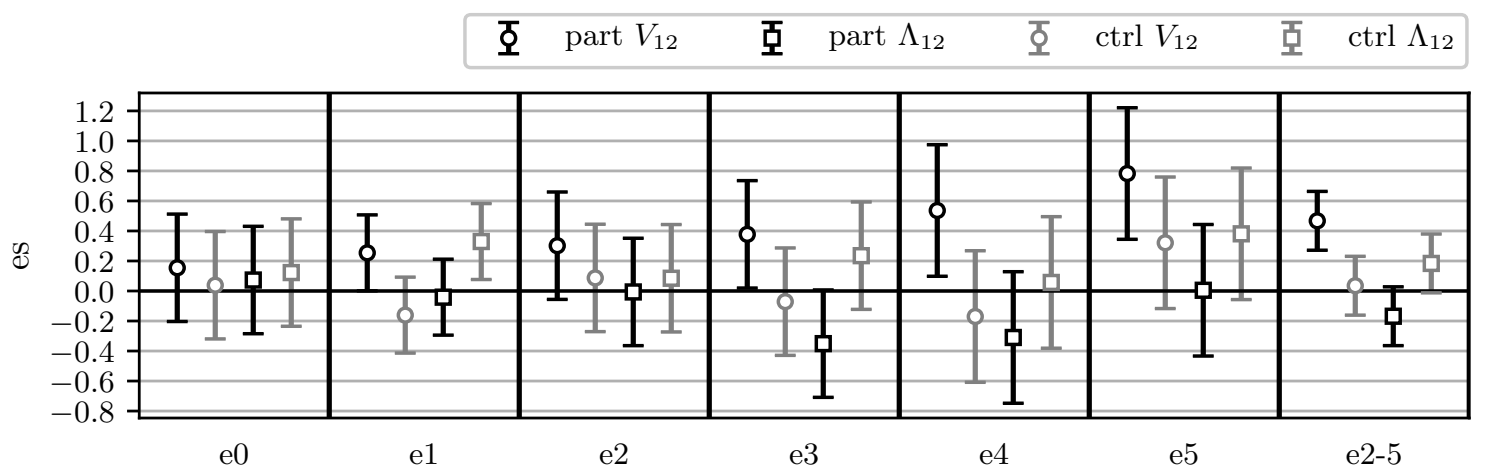

Figure 8: Effect sizes for the exploratory experiments. The error bars represent the $95 \%$ confidence interval, calculated as $1.96 / \sqrt{N}$, where $N$ is the experiment sessions number. Default and reverse scenarios are considered.

$2-5$, the first and second half data overall results for $V_{12}$ are $z_{p}^{1 s t}=2.61, z_{p}^{2 n d}=3.51$ for the participant sessions and $z_{c}^{1 s t}=0.32, z_{c}^{2 n d}=0.10$ for the controls. Significant results for both halves in the participant sessions indicate a consistent $\psi \phi$-action across the time.

In experiment 3, two CCD frames were collected in the $100 \mathrm{~ms}$ window. The first was used to provide the real-time feedback and the second was simply stored - no effort was made to process it or to inform the participant of its variations. This design was used to investigate the supposed $\psi \phi$-interaction characteristics; whether it depends exclusively on some information reaching the conscious agent or can be better understood as a kind of field interaction that could reach the undisplayed frame. The second frame result for $V_{12}$ is $z_{p}=1.88, z_{c}=-0.65$, revealing a similar, but slightly smaller, participant result than that obtained with the first frame analysis, thus favoring the second hypothesis.

\subsection{Formal experiments}

When examining the exploratory results, in particular, the large effect sizes seen in exps. 4 and 5 , important questions arise concerning the replicability of their results and the legitimacy of the chosen signs that compose the $V_{12}$ variable. To investigate those issues, four new experiments were conducted.

Experiments 6 and 8 used the same feedback configuration as exp. 4; while exps. 7 and 9 mirrored exp. 5 feedback strategy. The same exps. 4 and $5 V_{12}$ sign composition rules were predefined: $V_{12}=+V_{1}-V_{2}$ for exps. 6 and 8 ; and $V_{12}=-V_{1}-V_{2}$ for exps. 7 and 9 ; where $V_{1}\langle 5-15\rangle$ and $V_{2}\langle 19-25\rangle$ followed the standard scenario definition. The pre-registered analysis method for obtaining each session/variable $z$-score was the same used in the exploratory experiments.

Table 5 summarizes the statistical results obtained for the intention/relax differential analysis. For $V_{1}$ and $V_{2}$, statistical significance is found in participant data from exps. 7 and 9 . However, a sign inversion is seen in exps. 8 and 9 participant data. The cumulative $z$-score plots for $V_{12}$ presented in Fig. (9), highlights the sign inversion between the two $N=40$ blocks. While the combined exps. 8-9 is statistically significant, the global exp. 6-9 result, both in the participant and control data, conforms to the null hypothesis.

\subsection{Post hoc meta-analysis}

In retrospect, the directional hypothesis being tested in the formal experiments is tighter than the original motivation of the study. While the primary hypothesis was concerned with absolute differences between intention and relax epochs, the tests performed in the formal studies were implicitly merged with a secondary hypothesis that the $z$-signs would be strictly associated with 
Exp. 6

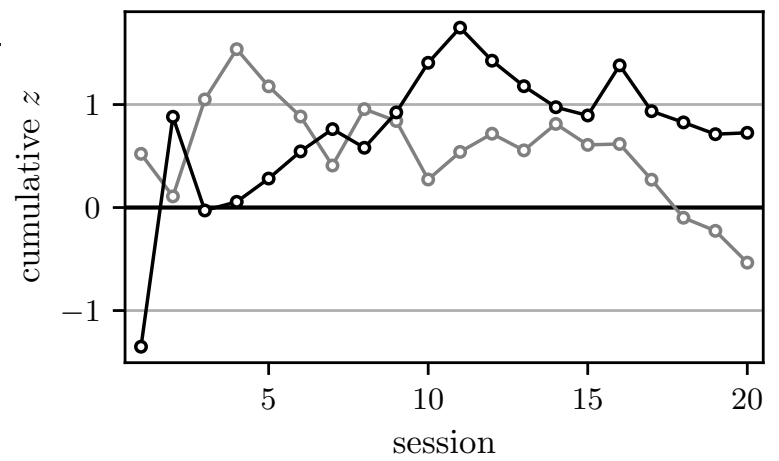

Exp. 8

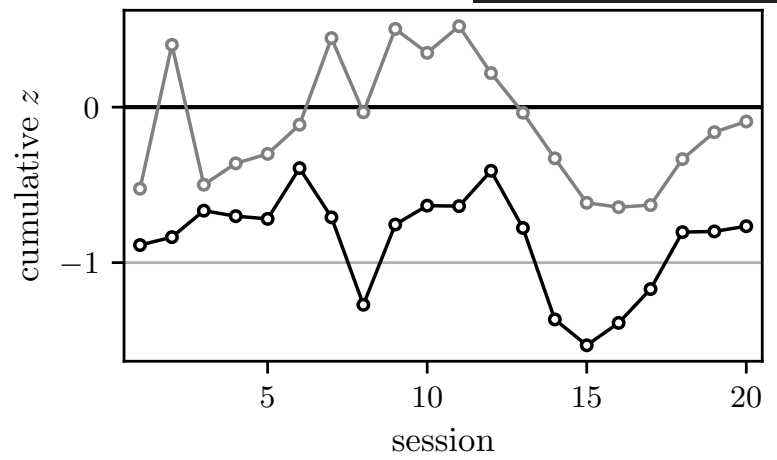

Exps. 6-7

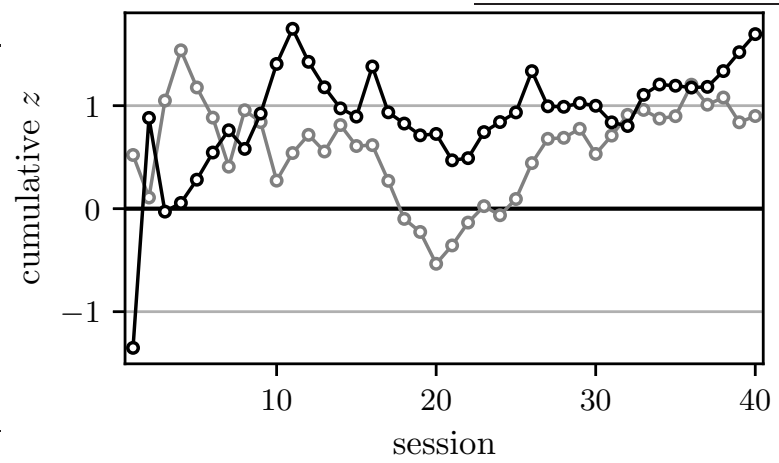

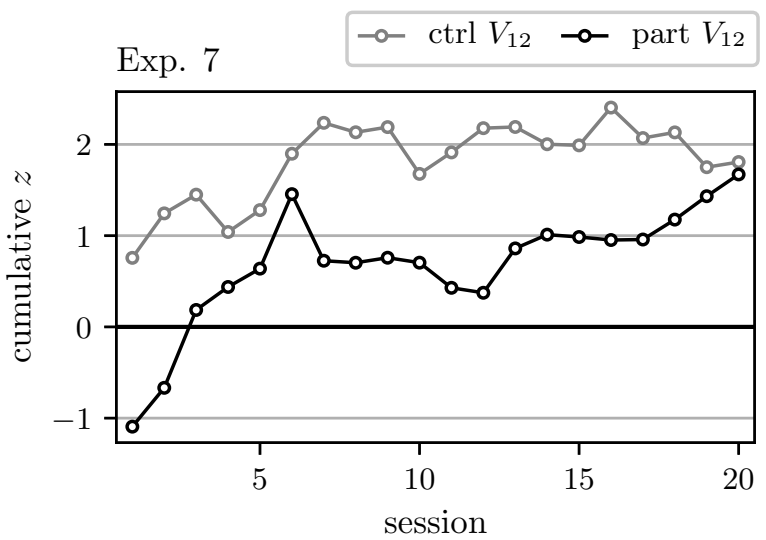

Exp. 9

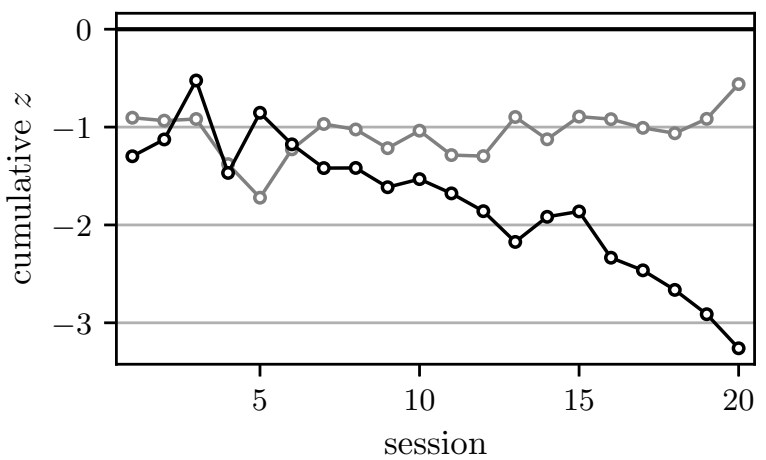

Exps. 8-9

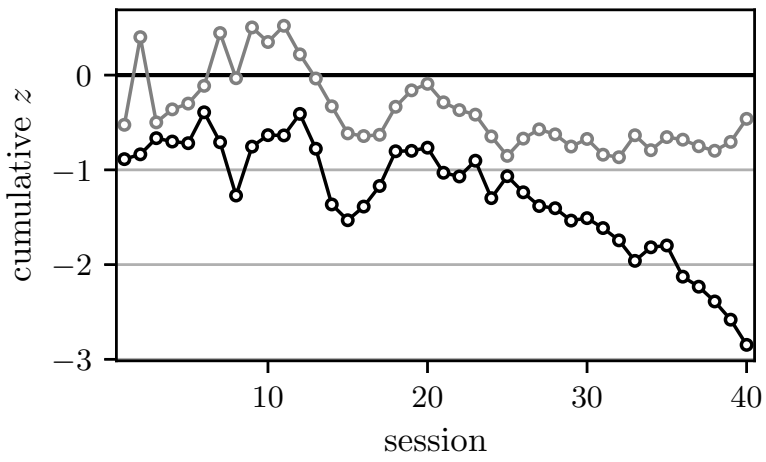

Exps. 6-9

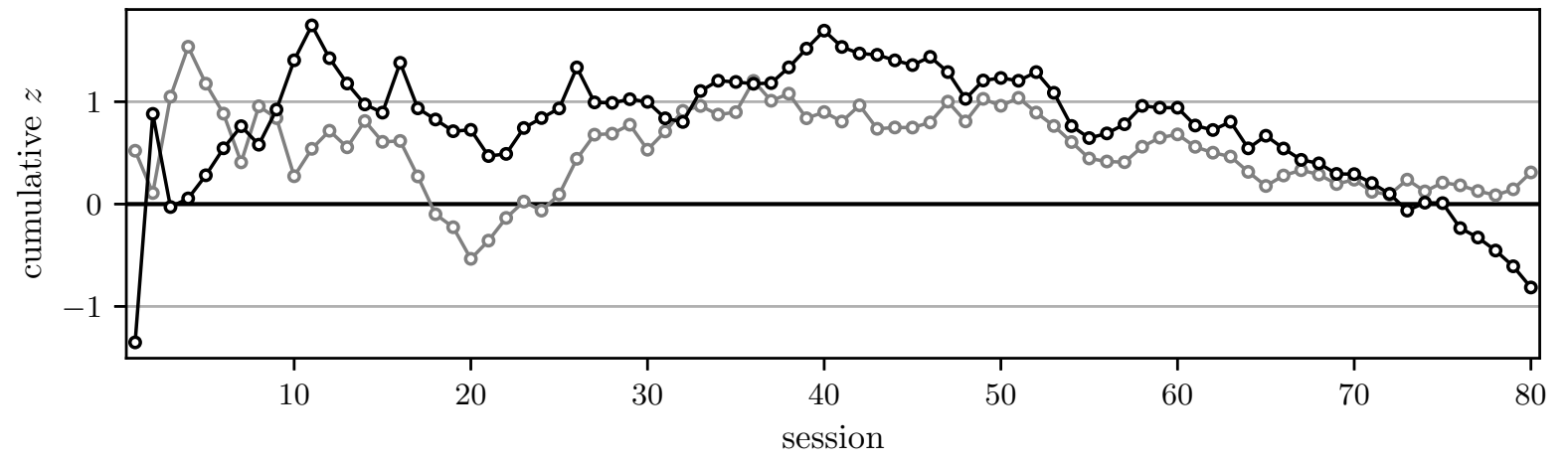

Figure 9: Cumulative z-score for the formal experiments. Cumulative plots are calculated as $\sum_{i=1}^{s} z_{i} / \sqrt{s}$, and given as a function of the session number $s$. The last points represent the values shown in Tab. (5). The bottommost plot reveals the global result for the 80 sessions from experiments $6-9$. 


\begin{tabular}{|cc|cc|cc|ccccc|}
\hline & & \multicolumn{2}{|c|}{$V_{1}\langle 5-15\rangle$} & \multicolumn{6}{|c|}{$V_{2}\langle 19-25\rangle$} & \multicolumn{6}{c|}{$V_{12}$} \\
\cline { 3 - 10 } exp. & $\mathrm{N}$ & $z_{p}$ & $z_{c}$ & $z_{p}$ & $z_{c}$ & comp. & $z_{p}$ & $z_{c}$ & $e s_{p}$ & $e s_{c}$ \\
\hline 6 & 20 & -0.22 & 0.54 & -0.22 & 1.71 & $+V_{1}-V_{2}$ & 0.73 & -0.54 & 0.16 & -0.12 \\
7 & 20 & -1.37 & -0.57 & -2.17 & -1.34 & $-V_{1}-V_{2}$ & 1.67 & 1.81 & 0.37 & 0.40 \\
8 & 20 & -1.53 & -0.87 & -0.30 & -0.41 & $+V_{1}-V_{2}$ & -0.77 & -0.09 & -0.17 & -0.02 \\
9 & 20 & 2.23 & -1.21 & 3.00 & 1.48 & $-V_{1}-V_{2}$ & -3.26 & -0.56 & -0.73 & -0.13 \\
$6-7$ & 40 & 0.81 & 0.78 & 1.69 & -0.27 & & 1.69 & 0.90 & 0.27 & 0.14 \\
$8-9$ & 40 & -2.66 & 0.24 & -1.91 & -0.75 & & -2.85 & -0.46 & -0.45 & -0.07 \\
$6-9$ & 80 & -1.31 & 0.72 & -0.16 & -0.72 & & -0.81 & 0.31 & -0.09 & 0.03 \\
\hline
\end{tabular}

Table 5: Formal experiments' results. The participant (control) sessions Stouffer's $z$-score is denoted as $z_{p}\left(z_{c}\right)$, while effect size is denoted as $e s_{p}\left(e s_{c}\right)$.

the feedback-favored slit. As a post hoc meta-analysis, the two hypotheses of difference and direction are decoupled into separate tests.

\subsubsection{Difference test}

The primary hypothesis is tested by applying Fisher's method to the experiments' results. According to this bi-directional method, the associated probabilities summed as $-2 \sum^{N_{e}} \ln p_{i}$ follow a chi-squared distribution with $2 N_{e}$ degrees of freedom, where $N_{e}$ describes the number of experiments being combined. Unlike with the Stouffer's method, the $z$-sign plays no role since a two-tailed probability yields the same value for $+z$ and $-z$.

The results for the Fisher combination are shown in Tab. (6) and Fig. (10), where the combined probabilities are converted back to standard scores. Statistical significance is found in the participant data, where all the controls conform to the null hypothesis.

\begin{tabular}{|c|cccc|cccc|cccc|}
\hline & \multicolumn{4}{|c|}{$V_{1}\langle 5-15\rangle$} & \multicolumn{4}{c|}{$V_{2}\langle 19-25\rangle$} & \multicolumn{4}{c|}{$V_{12}$} \\
exps. & $z_{p}$ & $z_{c}$ & $e s_{p}$ & $e s_{c}$ & $z_{p}$ & $z_{c}$ & $e s_{p}$ & $e s_{c}$ & $z_{p}$ & $z_{c}$ & $e s_{p}$ & $e s_{c}$ \\
\hline $2-5$ & 3.05 & 0.08 & 0.30 & 0.01 & 2.32 & 0.29 & 0.23 & 0.03 & 4.17 & 0.62 & 0.42 & 0.06 \\
$6-9$ & 1.95 & 0.63 & 0.22 & 0.07 & 2.53 & 1.59 & 0.28 & 0.18 & 2.75 & 0.72 & 0.31 & 0.08 \\
\hline
\end{tabular}

Table 6: Statistical results obtained with Fisher's method. $N_{e}$ is respectively 4 and 4 for exps. 2-5 and 6-9.

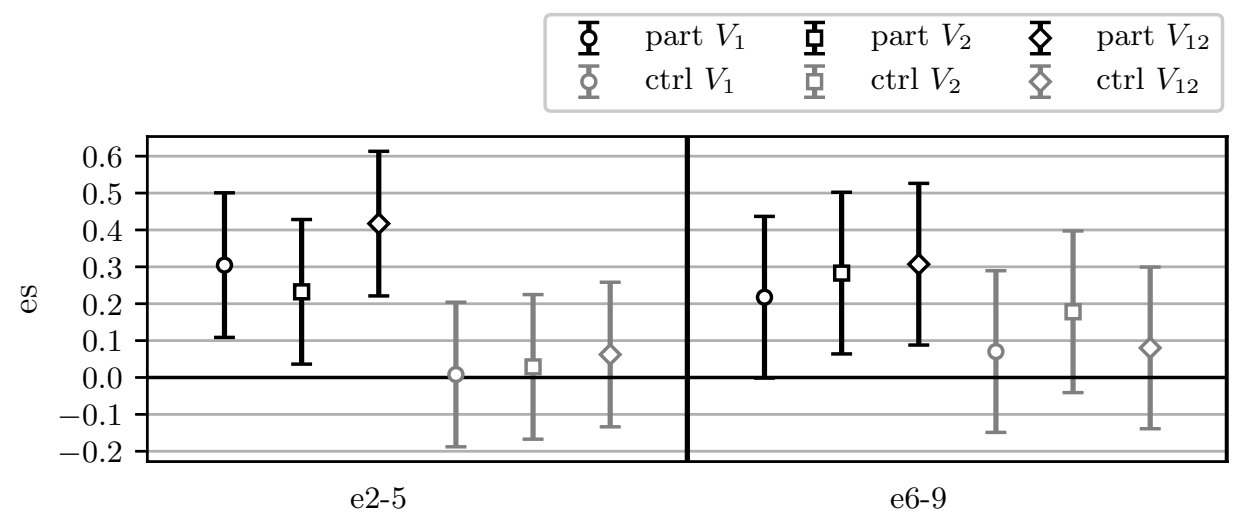

Figure 10: Effect sizes for experiments 2-5 and 6-9 combined with Fisher's method. The error bars represent the $95 \%$ confidence interval.

To achieve those results, the sessions' differential $z$-scores are first combined, as usual, into 
each experiment's Stouffer $z$-score; then, the experiments' $z$-scores are combined using Fisher's method. According to the literature, "the Stouffer test statistics is sensitive to consistent, even if mild, departures from null hypothesis in separate studies, whereas the Fisher procedure is most sensitive to occasional, extreme departures" [28, p. 66]. As stated, the use of a Stouffer combination within an experiment is strategic, aiming to catch small but consistent intention/relax differences in the same direction. If the direction can be consistently predicted by the feedback-favored slit, that's a secondary question detailed below.

\subsubsection{Direction test}

Two tests are performed to study the relationship between the obtained $z$-score signs and the feedback-favored slit: binomial and correlation. Both analyses use the $V_{1}$ and $V_{2} z$-scores obtained in exps. 4-9, since they all share the same feedback variable. Experiments 4,6,8 and $5,7,9$ are considered separately due to their opposite feedback-favored slit. The tests results are shown in Tab. (7).

\begin{tabular}{|c|cccc|cccc|cccc|}
\hline & \multicolumn{4}{|c|}{$V_{1}$ binomial } & \multicolumn{4}{c|}{$V_{2}$ binomial } & \multicolumn{4}{c|}{$V_{1} \& V_{2}$ correlation } \\
exps. & $n_{p}^{+}$ & $n_{c}^{+}$ & $p_{p}$ & $p_{c}$ & $n_{p}^{+}$ & $n_{c}^{+}$ & $p_{p}$ & $p_{c}$ & $r_{p}$ & $r_{c}$ & $p_{p}$ & $p_{c}$ \\
\hline $4,6,8$ & 26 & 27 & 0.25 & 0.37 & 27 & 31 & 0.37 & 0.70 & -0.32 & 0.04 & 0.01 & 0.74 \\
$5,7,9$ & 27 & 23 & 0.37 & 0.05 & 29 & 29 & 0.70 & 0.70 & 0.33 & -0.09 & 0.01 & 0.50 \\
\hline
\end{tabular}

Table 7: First two columns: binomial tests for the $V_{1}$ and $V_{2} z$-score signs. Third column: correlation between $V_{1}$ and $V_{2} z$-scores.

In the binomial analysis, the number of positive $z$-score sessions $n^{+}$is statistically evaluated according to a binomial distribution of $N=60$ and $p=0.5$. For $N=60$ sessions, a number of positive $z$ outcomes between 23 and 37 is expected as statistical fluctuation (with $\alpha=5 \%$ ). As seen in the results table, no significant outcomes were found for any condition.

For the correlation analysis, the Pearson's $r$ is obtained for the $V_{1}$ and $V_{2}$ differential zscores. Statistical significance is found for the participant data, providing interesting evidence for the $\psi \phi$-interaction; in terms of absolute $z$-score value, both variables tend to result in values of correlated magnitude, while the values found in the control data are uncorrelated. The results also show that the correlation signs are inverted between the two experimental groups, confirming the legitimacy of having used the $V_{12}$ sign compositions. As the scores are anti-correlated in exps. 4,6,8, the compound variable should follow the generic form of $V_{12}= \pm\left(V_{1}-V_{2}\right)$; and since in exps. 5,7,9 the scores are correlated, the variable is generically expressed as $V_{12}= \pm\left(V_{1}+V_{2}\right)$.

\subsection{Environment variables}

The differential analysis was also applied to the environment variables, and the resulting standard scores are presented in Tab. (8), where global scores are combined using Fisher's method. Two variables resulted in global statistical significance, the laser temperature $T_{L}$ and the CCD external temperature $T 2_{C}$. The latter one shows significance also for the control data, which is indicative of an artifact possibly related to analog-digital resolution and discrete temperature jumps.

To determine whether those two variables, or any other, could be associated with the differences measured in the $V_{1,2}$ variables of interest, the Pearson's $r$ correlation was calculated between the 240 differential $z$-scores obtained for the $V_{1,2}$ variables and the 240 differential $z$-scores obtained for the environmental variables. The CCD internal temperature $T_{C}$ vs $V_{1}$ was the only combination showing a statistically significant correlation in the participant data, with $r_{p}=-0.18, p_{p}=0.005$; and $r_{c}=0.04, p_{c}=0.49$ for the controls. 


\begin{tabular}{|c|cc|cc|cc|cc|cc|cc|cc|}
\hline \multirow{2}{*}{ exp. } & \multicolumn{2}{|c|}{$T_{C}$} & \multicolumn{2}{c|}{$T_{L}$} & \multicolumn{2}{c|}{$T_{R}$} & \multicolumn{2}{|c|}{$\left|M_{x}\right|$} & \multicolumn{2}{|c|}{$\left|M_{y}\right|$} & \multicolumn{2}{|c|}{$\left|M_{z}\right|$} \\
\cline { 2 - 12 } & $z_{p}$ & $z_{c}$ & $z_{p}$ & $z_{c}$ & $z_{p}$ & $z_{c}$ & $z_{p}$ & $z_{c}$ & $z_{p}$ & $z_{c}$ & $z_{p}$ & $z_{c}$ \\
\hline 0 & -0.08 & 0.41 & 0.22 & 0.12 & 0.88 & 0.24 & -1.19 & -0.30 & 0.30 & 0.46 & 2.22 & -0.67 \\
1 & 1.17 & -0.33 & -0.25 & 0.53 & -0.70 & -0.23 & 0.91 & 0.43 & -0.34 & -0.09 & 0.07 & 0.16 \\
2 & -0.69 & 0.91 & -1.21 & 1.49 & 1.03 & 0.12 & 0.19 & -0.23 & 0.70 & -0.10 & -0.83 & -1.36 \\
3 & -1.14 & 1.28 & 3.14 & -0.21 & -1.61 & 1.35 & -0.59 & -1.07 & -2.12 & 0.71 & 2.43 & -0.18 \\
4 & -0.73 & 0.03 & -0.68 & -0.22 & -0.15 & 0.18 & -0.05 & 1.26 & -0.10 & -0.04 & 0.70 & -0.70 \\
5 & -0.11 & 0.44 & 0.20 & -1.09 & -1.15 & 0.34 & -1.24 & -1.10 & -1.53 & 0.98 & -1.15 & -0.63 \\
6 & -0.92 & 0.68 & -0.66 & 0.32 & 0.01 & -0.34 & 0.69 & -0.46 & 0.18 & -0.94 & -0.12 & 0.10 \\
7 & 0.20 & 0.14 & -2.53 & -1.06 & -0.24 & -0.50 & -1.73 & 0.32 & 0.04 & 0.04 & 0.35 & -1.06 \\
8 & 0.47 & 0.49 & -1.85 & -1.61 & 1.36 & 0.87 & 0.35 & -1.04 & 0.60 & 0.09 & -0.01 & 0.97 \\
9 & 0.26 & -0.55 & -1.67 & -1.85 & -0.46 & 0.04 & -0.30 & 1.46 & 0.03 & 2.05 & 0.99 & -0.62 \\
$1-9$ & 0.22 & 0.10 & 2.93 & 1.12 & 0.55 & 0.04 & 0.37 & 0.67 & 0.41 & 0.25 & 0.71 & 0.26 \\
\hline
\end{tabular}

\begin{tabular}{|c|cc|cc|cc|cc|}
\hline \multirow{2}{*}{ exp. } & \multicolumn{2}{|c|}{$T 2_{C}$} & \multicolumn{2}{c|}{$\left|M 2_{x}\right|$} & \multicolumn{2}{|c|}{$\left|M 2_{y}\right|$} & \multicolumn{2}{|c|}{$\left|M 2_{z}\right|$} \\
\cline { 2 - 9 } & $z_{p}$ & $z_{c}$ & $z_{p}$ & $z_{c}$ & $z_{p}$ & $z_{c}$ & $z_{p}$ & $z_{c}$ \\
\hline 4 & -1.25 & 0.98 & 0.39 & -1.26 & 1.00 & -1.32 & 0.08 & -0.14 \\
5 & 0.19 & -0.58 & 0.60 & -0.21 & 1.54 & -0.67 & 0.27 & 0.67 \\
6 & -2.08 & 1.89 & 1.08 & -0.69 & 0.12 & -0.13 & 0.63 & -0.86 \\
7 & 2.16 & 2.37 & 0.05 & 1.23 & 0.73 & 0.71 & 0.14 & 0.44 \\
8 & 3.57 & 2.87 & -0.60 & 0.28 & -0.10 & -0.39 & -0.54 & -0.02 \\
9 & 4.34 & 4.46 & -0.49 & -0.03 & 0.25 & -0.08 & -0.76 & 0.54 \\
$4-9$ & 5.23 & 4.94 & 0.16 & 0.33 & 0.36 & 0.21 & 0.04 & 0.07 \\
\hline
\end{tabular}

Table 8: Differential $z$-scores for the environmental variables throughout the experiments. To investigate magnitude variations, the magnetic field components were transformed into their absolute values before analysis. Global scores are obtained with Fisher's method.

No environmental variable resulted in both a global significant $z$-score and a significant correlation to the $V_{1,2}$ differential $z$-score. This excludes the trivial explanation of temperature or magnetic field variations in intention/relax conditions being the primary cause of the changes measured in the $V_{1,2}$ variables.

\section{Discussion}

The exploratory experiments testing a consciousness-related form of interaction with a doubleslit system resulted in a highly significant difference between the intention and relax conditions. The subsequent formal experiments then tried to replicate these previous findings, failing to reach global significance. While one may feel compelled to straightforwardly interpret this result as the nonexistence of the investigated interaction, the following arguments challenge key aspects of this interpretation.

In the formal experiments, one may claim that some statistical fluctuations may have locally occurred in some experiments, but globally, and for a sufficiently large dataset, they converge to the expected null difference. This interpretation is challenged by the standard score magnitudes found in participant data as compared with the controls, as quantitatively revealed by the Fisher test performed in the post hoc analysis. One must have in mind that under the no-interaction scenario, participant and control data are understood as essentially equivalent.

A possible objection would be that the control and participant data cannot be equally 
classified because of the participant's bodily presence in the experimental room; hence heat, vibrations, and electromagnetic radiation could explain the larger effect sizes. While the body may slightly affect the measurements, it must be noted that the variables have their trends corrected with an $8^{\text {th }}$ order polynomial, and that the measured effect translates to a consistent difference between the detrended values obtained in the 40 alternated intention and relax epochs.

With regard to the physical mechanisms that could influence the participant data, heat is a monitored quantity, and no sensor resulted in both a globally significant differential score and a significant correlation to the variables of interest. Also, in experiment 0 a lamp producing more heat than a human body replaced the participant, demonstrating that a temperature increase in the experimental room cannot account for the measured effects. Vibrations and electromagnetic influences were highly attenuated with the respective use of a vibration isolation table and a Faraday's cage. Even in the case of minor leakages, the oscillatory nature of vibration is more likely to introduce noise into the measurements than a direction-consistent variation that could mimic a signal; and if the participant can (according to their intention) modulate a electromagnetic emission that affects the double-slit system, that itself indicates a novel technology.

According to the no-interaction interpretation, one must claim that the exploratory experiments' results are due to data dredging. The first response to this view is related to the physical meaning of the variables of interest. The variables explored by the optimization technique are theoretically justified by the mathematical model predicting the $\psi \phi$-interaction signatures according to the system's measured geometry. This is also important as it restricts the number of possible combinations, as opposed to a variable not bound to any physical meaning.

As a further challenge to the data-dredging argument, one faces again the participant/control equivalence; if both datasets are equivalent, how likely is one to find such a big effect in only one of them while applying the exact same analysis to both? It is reasonable to ask if the results could be somehow inflated, but in the absence of a legitimate interaction, the inflation should equally affect both samples. The final challenge is empirical, and presented by the so-called reverse study where the author tried to deliberately hack the control data in order to artificially produce the largest possible intention/relax differences - a task which failed to produce a statistically significant global effect.

In contrast, the post hoc meta-analysis results may be indicative of an anomalous interaction. The word meta is emphasized in the sense that it simply implements a different way of combining the experiments' global scores, where all the session scores are unaltered. The intent of this post hoc analysis was to decouple the formally tested hypothesis into two different tests: of an absolute difference between intention/relax conditions, and of a causal relationship between the difference sign and the slit targeted in the feedback. The bi-directional analysis revealed significant intention/relax absolute deviations in the participant data, while the relationship between the feedback-favored slit and the $V_{1,2}$ variables was found in their mutual correlation value rather than in their absolute signs.

One challenge faced by the anomalous interaction interpretation is in terms of meaning; if one cannot control the effect sign obtained, what is happening within the underlying physical process? A possible argument for the inability to control the light-enhanced slit is based on the discrepancy between the variable used to provide feedback and the one used in the offline analysis. While the first variable is built using the Fourier magnitude component, the second uses the phase. This difference is attributable to the author's learning curve during the study, and can thus be avoided in future studies. A more speculative explanation points to some sort of global conservation law underlying the phenomenon; while local significant differences may occur, globally and across time they tend to cancel out. Knowing this, future studies can pre-register to combine the experiment's results using the Fisher method.

The main obstacle for the $\psi \phi$-interaction interpretation is its controversial nature - the interaction shouldn't exist according to the current scientific world view. Also, if the interaction 
exists how could it have remained undetected given the technological breakthroughs of the last century? First, it's reasonable to expect a small cross-section; an effect so small that it would ordinarily go undetected, that needs a large dataset and proper amplification to be statistically detected in a controlled setting. Second, being a function of the conscious agent subjective condition, it may rely on a specific state of consciousness and sufficient skill, thus not being consistently achievable by anyone in any situation. In particular, if the effect happens to be catalyzed by states opposed to ordinarily prevailing rational faculties such as thinking and the use of language, a paradoxical situation may ensue; the more one tries to exert control, the less they cause the phenomenon. A third reason can be advanced as a consequence of the sociocultural process described by [29, Chap. 1] that led physicists to shift from philosophical interests to a more pragmatic approach in response to post-world war II military interests. While it was not uncommon for the founding fathers of quantum physics to discuss topics such as consciousness and mysticism, because of the post-war technological race, the interest in such topics not only became old-fashioned but something to be avoided while following a "serious"

career path. As a result, the current consensus holds that consciousness is not necessary to describe the physical world, while not introducing consciousness per se in their experiments.

More experiments should then be carried to formally test Fisher's combination and clarify the interpretation of the present results. In such experiments the feedback variable should be similar to the one used in the final analysis, and the two methods for combining the experiments' results should be pre-registered - Stouffer and Fisher. This protocol will more precisely test the secondary hypothesis for the relationship between the feedback-enhanced slit and the sign of the differential scores, as well as the reproducibility of the bi-directional effects.

Also, improvements to the current double-slit setup can be achieved through strategies such as the use of a Peltier cooled CCD sensor to improve the signal-to-noise ratio in the interference pattern measurements, and by implementing a real-time detrending method to provide more reliable feedback information. As to the study design, it's recommended that the experiment begin in an exploratory mode, and move to formal studies when sufficient knowledge is gained from the apparatus. To further rule out the participant's bodily presence as a possible artifact source, a non-local version of the experiment can be performed with participants situated outside of the experimental room when they receive the real-time feedback. From the theoretical side, refinements in the interaction model can be sought in order to provide even sharper variable predictions.

\section{Conclusion}

The four pre-registered experiments combined resulted in a statistically null difference between the data collected in intention and relax conditions. A post hoc combination of the formal experiments' scores using sign independent statistics, however, provided statistically significant results favoring the existence of anomalous interactions between conscious agents and a physical system. Further studies are warranted to formally test the post hoc hypothesis.

\section{Acknowledgements}

The author gratefully thanks all of the participants involved in this research for dedicating their time and attention to the experimental task. The author is also grateful to Dean Radin, for advice about conducting the experiment; Hartmut Grote, for helpful comments on earlier drafts of this manuscript; Wellington Zangari, for discussions on the subject matter; Mikiya Muramatsu, for discussions on the physical properties of the optical system; Diogo Soga and Ricardo Menegasso for technical support; and George Ortega and Grant Norrie, for language revision. 


\section{References}

[1] D. J. Chalmers, "Facing up to the problem of consciousness," Journal of consciousness studies, vol. 2, no. 3, pp. 200-219, 1995.

[2] J. Von Neumann, Mathematical foundations of quantum mechanics. No. 2, Princeton university press, 1955 .

[3] F. London and E. Bauer, "The theory of observation in quantum mechanics (1939)," in Quantum theory and measurement (J. A. Wheeler and W. H. Zurek, eds.), pp. 217-259, Princeton University Press, 1983.

[4] E. P. Wigner, "Remarks on the mind-body problem (1961)," in Quantum theory and measurement (J. A. Wheeler and W. H. Zurek, eds.), pp. 168-181, Princeton University Press, 2014 .

[5] H. P. Stapp, "Mind, matter, and quantum mechanics," Foundations of Physics, vol. 12, no. 4, pp. 363-399, 1982.

[6] E. J. Squires, "Quantum theory and the relation between the conscious mind and the physical world," Synthese, vol. 97, no. 1, pp. 109-123, 1993.

[7] B. Rosenblum and F. Kuttner, "The observer in the quantum experiment," Foundations of Physics, vol. 32, no. 8, pp. 1273-1293, 2002.

[8] B. d'Espagnat, "Consciousness and the wigner's friend problem," Foundations of Physics, vol. 35, no. 12, pp. 1943-1966, 2005.

[9] H. Margenau, "Measurements in quantum mechanics," Annals of Physics, vol. 23, no. 3, pp. 469-485, 1963.

[10] H. D. Zeh, "On the interpretation of measurement in quantum theory," Foundations of Physics, vol. 1, no. 1, pp. 69-76, 1970.

[11] S. Goldstein, "Quantum theory without observers - parts one and two," Physics Today, vol. 51, no. 3 and 4, pp. 42-46 and 38-42, 1998.

[12] M. Schlosshauer, J. Kofler, and A. Zeilinger, "A snapshot of foundational attitudes toward quantum mechanics," Studies in History and Philosophy of Science Part B: Studies in History and Philosophy of Modern Physics, vol. 44, no. 3, pp. 222-230, 2013.

[13] S. Yu and D. Nikolić, "Quantum mechanics needs no consciousness," Annalen der Physik, vol. 523, no. 11, pp. 931-938, 2011.

[14] D. I. Radin and R. D. Nelson, "Evidence for consciousness-related anomalies in random physical systems," Foundations of Physics, vol. 19, no. 12, pp. 1499-1514, 1989.

[15] H. Bösch, F. Steinkamp, and E. Boller, "Examining psychokinesis: the interaction of human intention with random number generators-a meta-analysis.," Psychological bulletin, vol. 132, no. 4, pp. 497-523, 2006.

[16] D. Radin, R. Nelson, Y. Dobyns, and J. Houtkooper, "Reexamining psychokinesis: comment on bösch, steinkamp, and boller (2006).," Psychological bulletin, vol. 132, no. 4, pp. 529-532, 2006.

[17] M. Ibison and S. Jeffers, "A double-slit diffraction experiment to investigate claims of consciousness-related anomalies," Journal of Scientific Exploration, vol. 12, no. 4, pp. 54350, 1998. 
[18] W. K. Wootters and W. H. Zurek, "Complementarity in the double-slit experiment: Quantum nonseparability and a quantitative statement of bohr's principle," Physical Review D, vol. 19 , no. 2 , p. $473,1979$.

[19] D. Radin, L. Michel, K. Galdamez, P. Wendland, R. Rickenbach, and A. Delorme, "Consciousness and the double-slit interference pattern: Six experiments," Physics Essays, vol. 25, no. 2, p. 157, 2012.

[20] D. Radin, L. Michel, J. Johnston, and A. Delorme, "Psychophysical interactions with a double-slit interference pattern," Physics essays, vol. 26, no. 4, pp. 553-566, 2013.

[21] D. Radin, L. Michel, A. Pierce, and A. Delorme, "Psychophysical interactions with a singlephoton double-slit optical system," Quantum Biosystems, vol. 6, no. 1, pp. 82-98, 2015.

[22] D. Radin, L. Michel, and A. Delorme, "Psychophysical modulation of fringe visibility in a distant double-slit optical system," Physics Essays, vol. 29, no. 1, pp. 14-22, 2016.

[23] A. Tellegen and G. Atkinson, "Openness to absorbing and self-altering experiences ("absorption"), a trait related to hypnotic susceptibility.," Journal of abnormal psychology, vol. 83, no. 3, p. 268, 1974.

[24] J. Goodman, Introduction to Fourier Optics. W. H. Freeman, third ed.

[25] S. Smith, Digital Signal Processing: A Practical Guide for Engineers and Scientists. Elsevier Science, 2003.

[26] G. Guerrer, "Pre-registration for experiments 6 and 7," Open Science Framework, registration form, 2017, http://dx.doi.org/10.17605/OSF.IO/6WXV5.

[27] G. Guerrer, "Pre-registration for experiments 8 and 9," Open Science Framework, registration form, 2017, http://dx.doi.org/10.17605/OSF.IO/9V4Z5.

[28] R. Abelson, Statistics As Principled Argument. Taylor \& Francis, 2012.

[29] D. Kaiser, How the hippies saved physics: science, counterculture, and the quantum revival. WW Norton \& Company, 2012. 


\section{Supporting Information: Consciousness-related interactions in a double-slit optical system}

\section{Appendix S1 Interaction signature properties}

This section explores how the interaction signatures described in Section 2.7 vary according to the sign of the $c$ parameter. To begin, a generic complex sum is considered for $z_{1,2}=r_{1,2} e^{i \theta_{1,2}}$, where $r$ and $\theta$ are real numbers. Their sum in polar coordinates is given by:

$$
z_{1}+z_{2}=\sqrt{r_{1}^{2}+r_{2}^{2}+2 r_{1} r_{2} \cos \left(\theta_{2}-\theta_{1}\right)} \exp \left\{i\left[\theta_{1}+\arctan \left(\frac{r_{2} \sin \left(\theta_{2}-\theta_{1}\right)}{r_{1}+r_{2} \cos \left(\theta_{2}-\theta_{1}\right)}\right)\right]\right\} \text {. }
$$

From this equation, it's possible to conclude that the compound magnitude component is invariant to an $r_{1} \leftrightarrow r_{2}$ exchange, while the compound phase is not.

In Section 2.7 the interaction signatures were obtained by a $\mathcal{F}$ Fourier transform applied to the interference pattern described in Eq. (5) . According to its linearity property, this particular transformation can be seen as a sum of three complex numbers. Since the third term doesn't depend on $c$, only the first two are considered in a complex sum with $M$ magnitude and $P$ phase:

$$
\frac{1+c \psi}{2} \mathcal{F}\left\{I_{1}\right\}(k)+\frac{1-c \psi}{2} \mathcal{F}\left\{I_{2}\right\}(k)=M(k, c, \psi) \exp [i P(k, c, \psi)] .
$$

The functions $I_{1}(x)$ and $I_{2}(x)$ represent the diffraction patterns produced by slits 1 and 2 . While spatially separated, they can have the same Fourier-transform magnitudes if their shapes are identical. In such a scenario, it's possible to show that $M(-c)=M(c)$, i.e. the impossibility of discriminating the specific slit 1 or 2 associated with a $\psi$ effect. The demonstration follows from using Eq. (S1) to obtain $M(k, c, \psi)$, and realizing that the $r_{1} \leftrightarrow r_{2}$ exchange corresponds to a $c$ sign inversion if the $I_{1}$ and $I_{2}$ magnitude components are identical.

Alternately, differences between $I_{1}$ and $I_{2}$ shapes can produce asymmetries that enable one to discriminate the specific slit 1 or 2 associated with a $\psi$ effect. The shape differences can be caused by two conditions: a) $s_{1} \neq s_{2}$, or, different slit widths; b) $U_{r} \neq 1$, or, a different amount of light crossing each slit. Condition b) is a consequence of a), but it can also be enhanced by small rotations of the double-slit plane. Both conditions are met for the current setup, as shown in Tab. (11). For $U_{r}<1$, one finds that $M(-c)<M(c)$, and, consequently, $M(-c)-M(0)<0$ while $M(c)-M(0)>0$. The last two inequalities, that are an approximation to Eq. (6) describing the difference signatures, show that it's possible to obtain positive or negative magnitude differences for a respective slit 1 or 2 light intensity enhancement.

For the $P(k, c, \psi)$ phase component, no asymmetries are necessary to discriminate between $\pm c$. As previously shown, the compound phase is naturally asymmetric to an $r_{1} \leftrightarrow r_{2}$ exchange. Thus even for identical magnitudes one obtains $P(-c)<P(c)$, and, consequently, the positive or negative differences for $\pm c$ shown in Fig. (44).

\section{Appendix S2 Phase variable discontinuities}

The method for transforming a CCD frame in a variable of interest was described in Section 2.9. The present section details the strategies for avoiding artificial discontinuities that may arise in the process.

When transforming a complex number from Cartesian to polar coordinates, the obtained phase angles are confined to a $-\pi \ldots \pi$ interval. If the phase value is close to $\pm \pi$, variation over time may consequently result in a $2 \pi$ discontinuity. It's usually possible to detect and fix the jumps using an unwrap algorithm, as shown in Fig. (S1).

However, as shown in Fig. (S2), sometimes the algorithm fails in fixing the discontinuities. This occurs when the unwrap fails to detect a jump as a consequence of noise added to the 

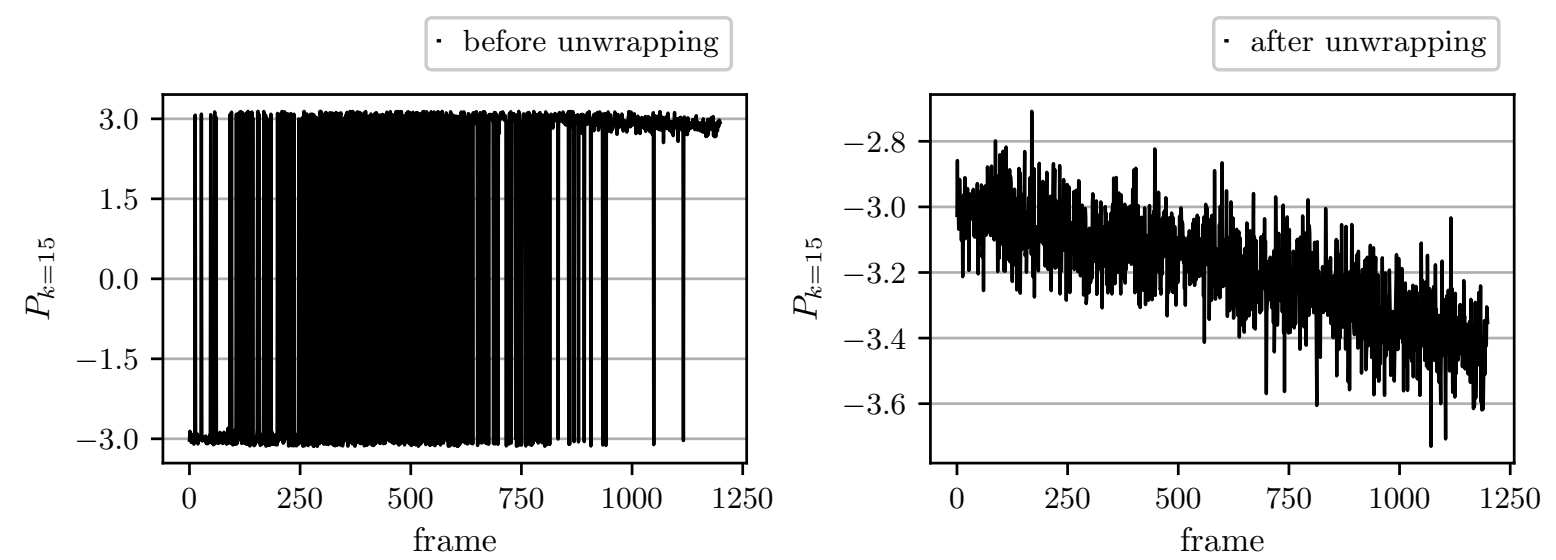

Figure S1: An example in the Fourier phase component at $k=15$ where unwrapping successfully fixes the $2 \pi$ discontinuities.

phase values. As discussed in the digital signal processing literature [25, p. 166], the smaller the magnitude associated with a certain $k$, the higher the phase noise. Thus, according to the magnitude values shown in Fig. (2), in the current setup one expects to find more unwrapsurviving discontinuities in $k$ between 10 and 40, than in $k$ between 1 and 9 .
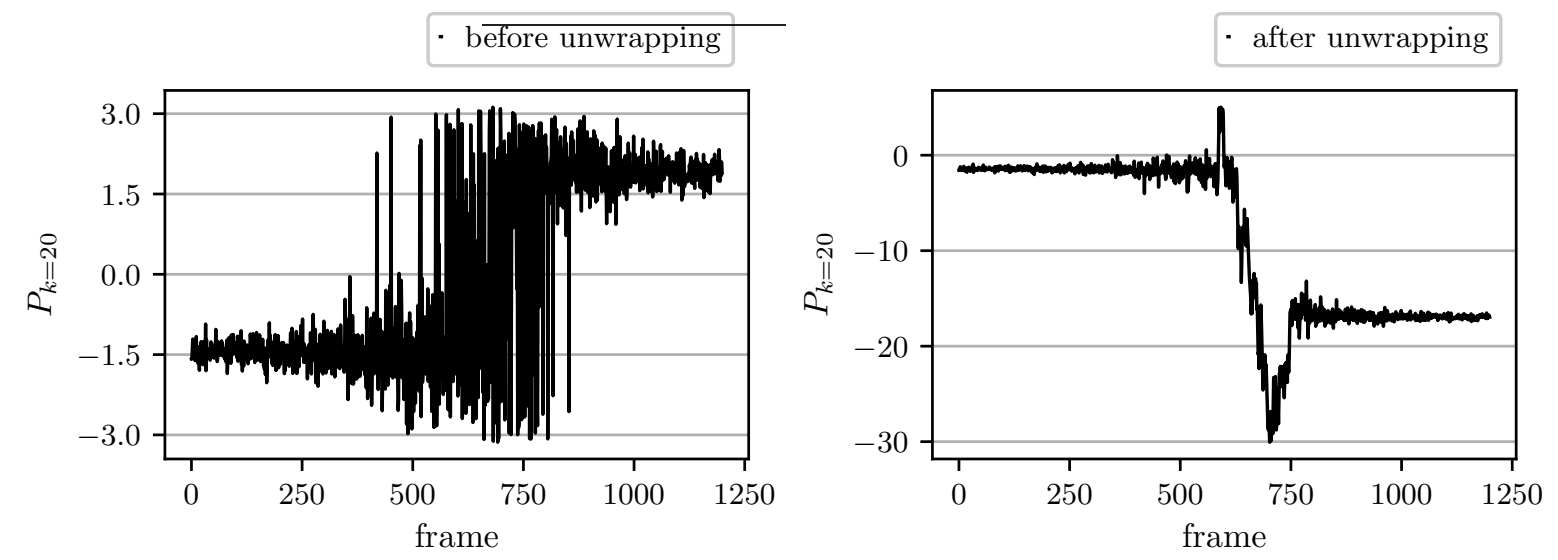

Figure S2: An example in the Fourier phase component at $k=20$ where unwrapping fails to fix the $2 \pi$ jumps, producing even larger discontinuities.

Discontinuities in the time series are bound to introduce artifacts in a differential analysis, and must therefore be avoided. When building the variable of interest, the standard deviation of the unwrapped phase is used as a criteria for the detection of discontinuities. If the phase's standard deviation associated with a given $k$ is above a cut value, the phase component at the given $k$ is not allowed to contribute in the phase sum that defines the variable of interest.

Figure S3 reveals the rate of phase rejection per session as a function of the $k$ values used in variables $V_{1}\langle 5-15\rangle$ and $V_{2}\langle 19-25\rangle$. The 240 participant and 240 control sessions from experiments 1-9 are used for obtaining the graphs. As previously predicted, all rejection ratios are below 30\%, and are higher for larger $k$ values. Although the participant data show slightly higher rejection rates, the control and participant data reveal similar rejection shapes.

Phase discontinuities present a challenge for the use of phase variables in the feedback real-time environment. For example, if the feedback variable used is $V_{1}\langle 5-15\rangle$, and a unwrapsurviving discontinuity is detected at some point in the $k=15$ component, then one reaches a dilemma; allowing this component to contribute to the next phase sum computations introduces discontinuities, but excluding the component does also. 

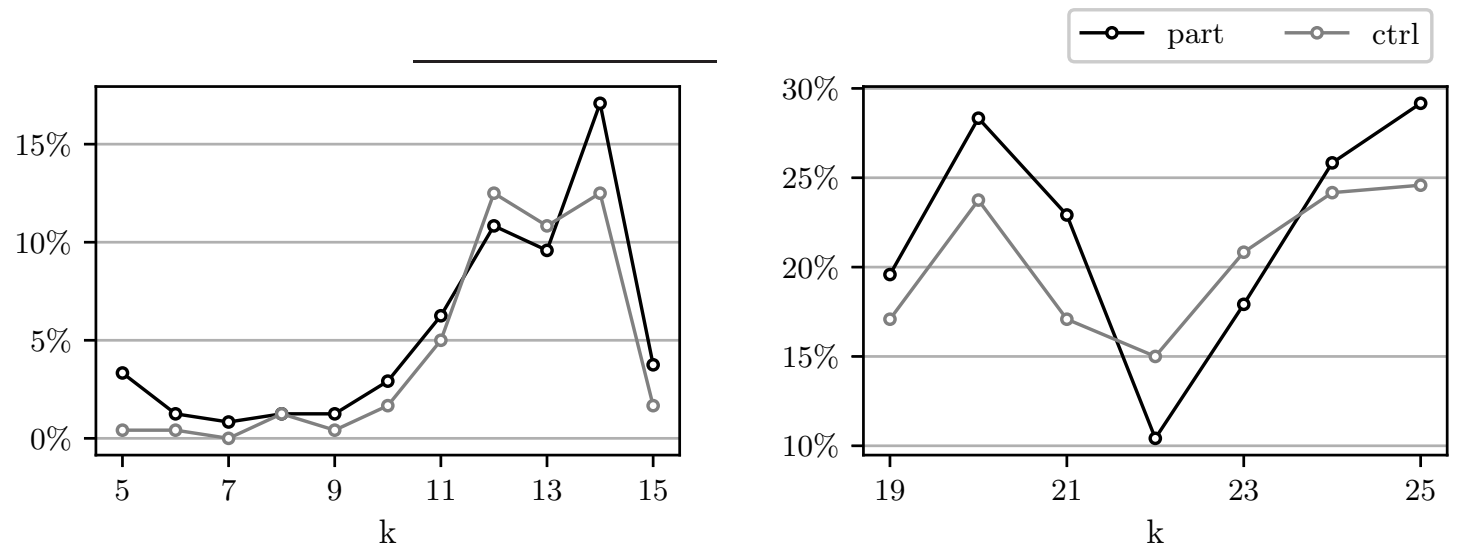

Figure S3: Phase discontinuity rejection per section for participant and control $N=240$ data. The rejection is associated with standard deviation values larger than 0.5. Left (right) plot show the rejection for $k$-values associated with the $V_{1}\left(V_{2}\right)$ variable. 